\title{
Energy Harvesting Communications With Batteries Having Cycle Constraints
}

\author{
Rajshekhar Vishweshwar Bhat ${ }^{\circledR}$, Member, IEEE, Mehul Motani ${ }^{\circledR}$, Fellow, IEEE, \\ Chandra R. Murthy ${ }^{\circledR}$, Senior Member, IEEE, and Rahul Vaze ${ }^{\circledR}$, Member, IEEE
}

\begin{abstract}
Practical energy harvesting (EH) based communication systems typically use a battery to temporarily store the harvested energy prior to its use for communication. The battery capacity can quickly degrade with time if it is subject to repeated shallow charge-discharge cycles. This motivates the cycle constraint which mandates that a battery must be charged only after it is sufficiently discharged and vice versa. We consider a Bernoulli energy arrival model, and a half-duplex battery constraint. In this context, we study EH communication systems with: (a) a single battery with capacity $2 B$ units and (b) dual batteries, each having capacity of $B$ units. The aim is to obtain the best possible long-term average throughputs in point-to-point (P2P) channels and multiple access channels (MAC). For the P2P channel, we obtain an analytical optimal solution in the single battery case, and propose optimal and suboptimal power allocation policies for the dual battery case. We extend these policies to obtain achievable throughput regions in MACs by jointly allocating rates and powers. From numerical simulations, we find that the optimal throughput in the dual battery case can be more than twice of that in the single battery case, although the total energy storage capacity in both cases is $2 B$ units.
\end{abstract}

Index Terms-Energy harvesting, battery cycle and halfduplex constraints, single and dual battery architectures, Communication systems.

\section{INTRODUCTION}

$\mathbf{E}$ NERGY harvesting (EH) from natural and man-made sources has been envisioned as a viable technique for enabling low-power and energy starved communication systems, including Internet of Things (IoT) devices in fifth generation $(5 \mathrm{G})$ networks [2]. Consequently, EH communications has been widely studied in the last few years [3]-[6]. In most of the existing studies, the proposed power allocation policies may require batteries to undergo repeated partial

Manuscript received January 12, 2019; revised June 12, 2019 and August 1, 2019; accepted August 6, 2019. Date of publication September 4, 2019; date of current version March 18, 2020. The work of C. R. Murthy was supported by the Young Faculty Research Fellowship from the Ministry of Electronics and Information Technology, Government of India. Part of this work has been presented at the IEEE ICC 2019, Shanghai, China [1]. The associate editor coordinating the review of this article and approving it for publication was J. Yang. (Corresponding author: Rajshekhar Vishweshwar Bhat.)

R. V. Bhat is with the Department of Electrical Engineering, Indian Institute of Technology, Dharwad 580011, India (e-mail: rajshekhar.bhat@iitdh.ac.in).

M. Motani is with the Department of Electrical and Computer Engineering, National University of Singapore, Singapore 119077.

C. R. Murthy is with the Department of Electrical Communication Engineering, Indian Institute of Science, Bengaluru 560012, India.

R. Vaze is with the School of Technology and Computer Science, Tata Institute of Fundamental Research, Mumbai 400005, India.

Digital Object Identifier 10.1109/TGCN.2019.2939285 (shallow) charge and discharge cycles. In practice, such a charging and discharging pattern can quickly degrade the usable capacity of batteries. For instance, the usable capacity of many Nickel based batteries [7] and some Li-ion [8] batteries reduces significantly when they are subjected to shallow charge/discharge cycles. This phenomenon, referred to as the memory effect or voltage depression, can be avoided by imposing the so-called cycle constraint, i.e., by discharging (charging) the battery to a lower (an upper) limit before charging (discharging) it again [9]. Further, the practical batteries cannot be charged and discharged simultaneously, which we call as the half-duplex battery constraint. Hence, in this work, we consider the design of EH communication systems under (i) the cycle constraint, and (ii) the half-duplex battery constraint.

Although EH communications has received a significant attention, the impact of the cycle and half-duplex constraints of practical batteries has not been widely studied in the existing literature [3]. In a P2P channel, the authors in [10]-[12] consider the interplay between the battery charging and discharging policy and the irreversible degradation (aging) of its storage capacity. This irreversible degradation is different from the voltage depression; the latter can be avoided by the cycle constraint. In [13], the authors indirectly control the battery degradation by constraining the number of charge and discharge cycles per unit time, but their model does not explicitly account for the impact of the number of charge and discharge cycles on the battery capacity. In [14], a Bernoulli EH model is assumed, where, in a slot, either an energy packet with energy quantum equal to the capacity of the battery arrives, or no energy arrives. This implies, whenever a packet of energy arrives, the battery fills up completely. In this case, when a fresh energy packet arrives, the residual energy in the battery can be thought to be discarded instantaneously before replenishing it with the energy that arrived. Hence, [14] implicitly accounts for the cycle constraint since the battery has unit capacity. Based on the optimal online policy for the above Bernoulli EH model, the authors in [14] obtain a simpler fixed-fraction policy (FFP) which consumes a fixed fraction of the available energy in the battery in each slot. They obtain bounds on the absolute difference between and the ratios of the maximum achievable throughput of the FFP and that of the optimal policy with an infinite-capacity battery. This work has also been extended to multi-user settings in [15]-[17]. Recently, [18] generalized the approach developed in [14], to work for concave monotonically increasing utility functions of the transmit power in the single-user case. In this work, we 
consider a Bernoulli energy arrival model, similar to [19]-[21]. We study a case where multiple energy arrivals are needed to fill the battery. In addition to the cycle constraint, which has been implicitly assumed in [14]-[18], we also account for the practical half-duplex battery constraint (see [22], [23]). In this setting, for the single battery case, we obtain a simple optimal online policy, and for the dual battery case, we obtain a simple near-optimal online policy, similar to the FFP, with a constant additive gap from the maximum achievable throughput with an infinite-capacity battery.

Our goal is to obtain power policies that maximize the longterm average throughputs and throughput regions in single battery and dual battery cases in a P2P channel and a MAC under (i) the cycle constraint, and (ii) the half-duplex battery constraint. We assume that the throughput is a concave function of the transmit power. Due to the above constraints, in the single battery case, when the battery is being discharged (charged), energy harvesting (transmission) is suspended, and when the battery gets empty (full), charging (transmission) starts. Hence, if the energy from the battery is utilized aggressively, the duration of transmission will be short, implying that the time duration over which energy harvesting is suspended is short. However, due to the concavity, the time-averaged throughput in an aggressive policy could be less than that with a conservative policy with a slower energy utilization. Similarly, in the dual battery case, when a battery is being charged, the transmitter draws power from the other battery and the roles of the batteries are switched when the charging battery becomes full and the other battery gets empty. In this case, an aggressive policy leads to a lower throughput, as the working battery may get drained long before the charging battery gets full. However, a conservative policy may result in energy overflow, as the battery being discharged may not be empty when the charging battery becomes full. Between these two extremes lies the optimal solution. The main contributions of the paper are:

- In a P2P channel under the single battery case, we obtain an analytical solution to the long-term average throughput maximization problem in the online case with causal knowledge of energy arrivals.

- For a P2P channel under the dual battery case, we first obtain optimal power allocations via dynamic programing and then propose non-adaptive online policies, which do not exploit knowledge of current battery states, and adaptive policies, which adapt power allocations based on battery states when a new energy arrival occurs.

- For a $U$-user MAC, we derive long-term average achievable throughput regions in the single battery and dual battery cases, based on the online policies proposed for the P2P channel.

- Using numerical simulations, we show that the performance gap between the optimal policy for an ideal system equipped with an infinite-capacity battery and a proposed non-adaptive policy decays faster than the inverse of the square root of the battery capacity. Further, the largest throughput region in the single battery case is contained within that of the dual battery case.

In summary, our study finds that, under the cycle and half-duplex battery constraints, the optimal performance in the dual battery case is significantly better than that in the single battery case, although the total storage capacity in both cases is the same.

The remainder of the paper is organized as follows. The system model is presented in Section II. We study the P2P channel under single battery and dual battery cases in Sections III and IV, respectively. We then consider a MAC in Section V. Numerical results are presented in Section VI, followed by concluding remarks in Section VII.

\section{SySTEM MODEL}

We consider a P2P channel and a MAC under two cases: (a) the single battery case, in which the transmitters are equipped with a single battery having storage capacity of $2 B$ units, and (b) the dual battery case, in which the transmitters are equipped with two batteries, each having storage capacity of $B$ units. In both the cases, the transmitters are powered from EH sources. Further, the receiver is connected to the mains, and hence the receiver can always remain on. Now, based on our discussion in the introduction, we impose the half-duplex battery constraint, and the cycle constraint due to which the battery must be discharged (charged) to a lower limit, $C_{\min }$ (an upper limit, $C_{\max }$ ) before charging (discharging) it again, with $C_{\min }<C_{\max }$. Furthermore, the battery cannot be discharged below $C_{\min }$ or charged above $C_{\max }$. In this case, it is clear that $C_{\min }$ plays the same role as 0 units of energy in the battery, and $C_{\max }$ plays the role of the battery capacity. Hence, without loss of generality, we assume $C_{\min }=0$ for both the single battery and dual battery cases, and $C_{\max }=2 B$ and $C_{\max }=B$ for the single battery and dual battery cases, respectively.

\section{A. EH Model}

We consider a time-slotted system with unit slot length. The harvested energy arrives continuously at a constant rate in a slot such that the total amount of energy accumulated from the beginning of slot $i$ until the end of the slot is $E_{i}$ units, where $E_{1}, E_{2}, \ldots$ is a sequence of i.i.d. random variables, distributed as $E$, a non-negative random variable with mean $\mu$. As in [19]-[21], we adopt the following Bernoulli EH model:

$$
E= \begin{cases}E_{H} & \text { w.p. } p \\ 0 & \text { w.p. } 1-p,\end{cases}
$$

where w.p. stands for "with probability". In this case, the average EH rate, $\mu=p E_{H}$. We also assume $B$ and $E_{H}$ are related as $B / E_{H}=r$ for some integer $r \geq 1$.

\section{B. Energy Management and Evolution of Battery States}

The harvested energy is stored in a battery before using it for transmission.

1) Single Battery Case: In the single battery case in Fig. 1a, when the battery is being charged, no transmission is carried out. When transmission occurs, the energy harvesting is suspended, as batteries cannot be charged and discharged simultaneously. Hence, when $S_{1}\left(S_{2}\right)$ is closed, $S_{2}\left(S_{1}\right)$ will be open in Fig. 1a. Let $B_{i}$ be the amount of energy stored in the battery at the start of slot $i, P_{i}$ be the transmit power 


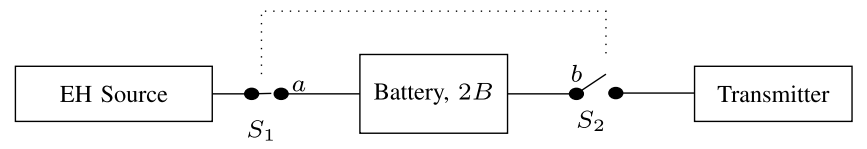

(a) The single battery case.

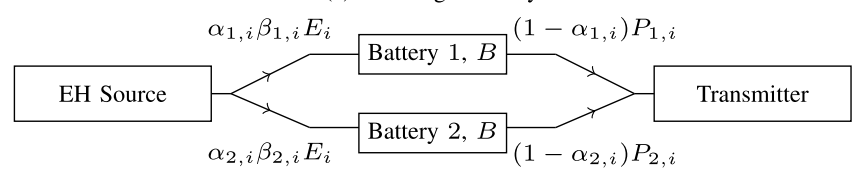

(b) The dual battery case.

Fig. 1. We consider EH users equipped with (a) single battery with capacity of $2 B$ units and (b) two batteries, each with capacity of $B$ units.

in slot $i$ and $\alpha_{i} \in\{0,1\}$ be an indicator variable, where $\alpha_{i}=1\left(\alpha_{i}=0\right)$ indicates that the battery is being charged (discharged) in slot $i$. Then, the battery evolves as follows:

$$
\begin{aligned}
& B_{i+1}=\max \left(\min \left(B_{i}+\alpha_{i} E_{i}, 2 B\right)-\left(1-\alpha_{i}\right) P_{i}, 0\right), \\
& \alpha_{i+1}=\alpha_{i} \mathbb{1}_{(0,2 B)}\left(B_{i+1}\right)+\mathbb{1}_{\{0\}}\left(B_{i+1}\right),
\end{aligned}
$$

for all $i \in\{1,2, \ldots$,$\} , where \mathbb{1}_{\mathcal{A}}(x) \triangleq 1$ if $x \in \mathcal{A}$ and $\mathbb{1}_{\mathcal{A}}(x) \triangleq 0$ if $x \notin \mathcal{A}$. Since we multiply $E_{i}$ and $P_{i}$ by $\alpha_{i}$ and $\left(1-\alpha_{i}\right)$, respectively, (2) captures the half-duplex constraint. Moreover, as $\alpha_{i}$ changes its value from 1 to 0 ( 0 to 1 ) only when the battery becomes full (empty), (3) accounts for the cycle constraint. Since we cannot use more energy than what is available in the battery, we also have the following constraint:

$$
P_{i} \leq 2 B_{i}, \quad i=1,2, \ldots
$$

2) Dual Battery Case: In the dual battery case in Fig. 1b, let $B_{j, i}$ be the amount of energy stored in battery $j \in\{1,2\}$ at the start of slot $i, P_{j, i}$ be the power drawn from battery $j$ in slot $i$ and $\alpha_{j, i} \in\{0,1\}$ be an indicator variable, where $\alpha_{j, i}=1\left(\alpha_{j, i}=0\right)$ indicates that the battery $j$ is being charged (discharged) in slot $i$. Further, let $\beta_{j, i} \geq 0$ be the fraction of the harvested energy used for charging battery $j$ in slot $i$ with $\beta_{1, i}+\beta_{2, i}=1$. The batteries evolve as follows:

$$
\begin{aligned}
& B_{j, i+1}=\max \left(\min \left(B_{j, i}+\alpha_{j, i} \beta_{j, i} E_{i}, B\right)-\left(1-\alpha_{j, i}\right) P_{j, i}, 0\right), \\
& \alpha_{j, i+1}=\alpha_{j, i} \mathbb{1}_{(0, B)}\left(B_{j, i+1}\right)+\mathbb{1}_{\{0\}}\left(B_{j, i+1}\right),
\end{aligned}
$$

for all $i \in\{1,2, \ldots\}$ and $j \in\{1,2\}$, where $P_{j, i} \leq B_{j, i}$. As in the single battery case, (5) and (6) account for the half-duplex and the cycle constraints, respectively. The total transmit power in slot $i$ is given by

$$
P_{i}=P_{1, i}\left(1-\alpha_{1, i}\right)+P_{2, i}\left(1-\alpha_{2, i}\right) .
$$

The min and max in the above equations capture the facts that the battery energy cannot exceed its capacity or become negative, respectively. In the rest of the work, we refer to the battery that is being charged (discharged) as the charging (working) battery.

\section{Throughput Maximization and an Upper Bound}

The communication is over an additive white Gaussian noise (AWGN) channel with unit noise power. We assume that the throughput with received signal-to-noise ratio of $P$ W in a slot is given by $R=\frac{1}{2} \log (1+P)$ bits-per-second (bps). All the logarithms are to the base 2 . Now, the long-term average throughput is defined as follows:

$$
T\left(P_{1}, P_{2}, \ldots\right) \triangleq \liminf _{k \rightarrow \infty} \frac{1}{k} \mathbb{E}\left[\sum_{i=1}^{k} \frac{1}{2} \log \left(1+P_{i}\right)\right],
$$

where the expectation is over all the possible sequences of energy arrivals. Our objective is to find the optimal power policy, $P_{1}, P_{2}, \ldots$, that maximizes the long-term average throughput, $T$ in (8), i.e., to solve the following optimization problems:

$$
\underset{P_{1}, P_{2}, \ldots}{\operatorname{maximize}} T\left(P_{1}, P_{2}, \ldots\right) \text { subject to }(2)-(4),
$$

for the single battery case, and

$$
\underset{P_{1}, P_{2}, \ldots}{\operatorname{maximize}} T\left(P_{1}, P_{2}, \ldots\right) \text { subject to }(5)-(7),
$$

for the dual battery case.

In order to benchmark the proposed power allocation policies, we now present an upper bound on $T$. When the cycle and half-duplex battery constraints are not present, and the users are equipped with infinite-capacity batteries, it is optimal to utilize $\epsilon$ smaller amount of power per slot on average, compared to the average harvesting rate, where $\epsilon>0$ can be arbitrarily small. Further, by the concavity of the rate function, it is optimal to utilize an equal amount of energy $\mu$ in every slot, resulting in the long-term average throughput [24]-[26]

$$
T_{\mathrm{ub}}=\frac{1}{2} \log (1+\mu)
$$

The subscript $u b$ indicates that this performance is an upper bound on the throughput obtainable under the half-duplex and the cycle constraints.

\section{P2P Channel: Single Battery Case}

In this section, we consider a P2P channel under the single battery case and obtain the optimal solution to the long-term average throughput maximization problem in (9). Let the number of slots required to accumulate at least $2 B$ units of energy in the battery be $L_{2 \mathrm{~B}}$, i.e.,

$$
L_{2 \mathrm{~B}} \triangleq \min \left(l: \sum_{i=1}^{l} E_{i} \geq 2 B\right) .
$$

Clearly, $L_{2 \mathrm{~B}}$ is a random variable. Let $\bar{L}_{2 \mathrm{~B}}$ be the expected value of $L_{2 \mathrm{~B}}$, i.e., $\bar{L}_{2 \mathrm{~B}} \triangleq \mathbb{E}\left[L_{2 \mathrm{~B}}\right]$. With the $\mathrm{EH}$ model in (1), since the energy inter-arrival times are i.i.d. geometrically distributed random variables with mean $1 / p$, we have $\bar{L}_{2 \mathrm{~B}}=2 r / p$. To obtain the optimal long-term average throughput, we assume that the initial energy stored in the battery is $2 B$ units. We then discharge and charge the battery subject to the half-duplex and the cycle constraints in (2) and (3), respectively. Consider the following randomized power allocation policy in which, when the battery gets full, we consume $2 B$ units of energy in $N$ slots, where $N=n$ w.p. $p_{n}$ such that $\sum_{n=1}^{\infty} p_{n}=1$ and $p_{n} \geq 0$ for all 
$n \in\{1,2, \ldots\}$. In these slots, the transmit power is kept constant at $2 B / N$ units. Note that, conditioned on $N=n$, it is optimal from a throughput perspective to divide the energy equally among the $n$ slots, due to the concavity of the $\log (\cdot)$ function. Starting from the $(n+1)^{\text {th }}$ slot, the battery is charged for $L_{2 \mathrm{~B}}$ slots to fill it, during which time no transmission occurs. Clearly, the $\left(n+L_{2 \mathrm{~B}}+1\right)^{\text {th }}$ slot is a renewal instant. Hence, the total reward and the length of the renewal period are $(N / 2) \log (1+2 B / N)$ bps and $\left(N+L_{2 \mathrm{~B}}\right)$ slots, respectively. Therefore, by the renewal-reward theorem [27] and (8), the long-term average throughput in the single battery case is equal to $T_{\mathrm{SB}}\left(p_{1}, p_{2}, \ldots\right)=\mathbb{E}\left[\frac{N}{2} \log \left(1+\frac{2 B}{N}\right)\right] / \mathbb{E}\left[N+L_{2 \mathrm{~B}}\right]=$ $\left(\sum_{n=1}^{\infty} p_{n} \frac{n}{2} \log \left(1+\frac{2 B}{n}\right)\right) /\left(\sum_{n=1}^{\infty} n p_{n}+\bar{L}_{2 \mathrm{~B}}\right)$. Now, the longterm average throughput maximization problem in (9) can be re-written as

$$
\underset{p_{n} \geq 0, n \in \mathbb{Z}^{+}}{\operatorname{maximize}} T_{\mathrm{SB}}\left(p_{1}, p_{2}, \ldots\right) \text { subject to } \sum_{n=1}^{\infty} p_{n}=1 \text {. }
$$

We present an equivalent optimization problem to (12) in the following lemma.

Lemma 1: The optimal long-term average throughput in the single battery case, obtained by solving (12), is equal to

$$
T_{\mathrm{SB}}^{*}=\max _{n \in \mathbb{Z}^{+}} \frac{\frac{n}{2} \log \left(1+\frac{2 B}{n}\right)}{n+\bar{L}_{2 \mathrm{~B}}},
$$

and the optimal distribution of $N$ is given by $p_{n^{*}}=1$ and $p_{n}=0, \forall n \neq n^{*}$, where $n^{*}$ is the value of $n$ that solves (13).

Proof: See Appendix A.

Due to Lemma 1, it suffices to solve (13) to obtain the optimal solution to (12). Note that (13) is an integer program. To solve it, we first allow $n$ to take any positive real value and solve the relaxed problem. We then optimally round the solution to satisfy the integer constraint in (13).

1) Relaxation: In the relaxed problem, we denote the transmit power and number of transmission slots by $\tilde{P}$ and $\tilde{n}$, respectively. Hence, we have, $\tilde{n}=2 B / \tilde{P}$ and the objective function in (13) becomes $\mu \log (1+\tilde{P}) /(2(\mu+\tilde{P}))$, where the average $\mathrm{EH}$ rate, $\mu=p E_{H}$ under the assumption that $r \geq 1$, i.e., $B \geq E_{H}$. Therefore, the optimal transmit power can be obtained by solving

$$
\max _{\tilde{P} \in \mathbb{R}^{+}} \frac{\mu \log (1+\tilde{P})}{2(\mu+\tilde{P})} .
$$

We now have the following theorem.

Theorem 2: The optimal solution to (14) is given by

$$
\tilde{P}^{*}=\exp (1) \exp \left(W_{0}(\exp (-1)(\mu-1))\right)-1 \text {, }
$$

and the optimal long-term average throughput is given by

$$
\tilde{T}_{\mathrm{SB}}=\frac{\mu}{2 \ln 2 \exp (1) \exp \left(W_{0}(\exp (-1)(\mu-1))\right)},
$$

where $W_{0}(\cdot)$ is the principal branch of the Lambert $\mathrm{W}$ function [28].

Proof: See Appendix B.
2) Rounding: From Theorem 2, clearly, $\tilde{n}^{*}=2 B / \tilde{P}^{*}$. Noting that $\tilde{n}^{*}$ may not be an integer, we now obtain the optimal solution to (13), with the integer constraint on $n$. From the proof of Theorem 2, recall that the first order derivative of the objective function has only a single positive real root, $\tilde{P}^{*}$ at which it attains the maximum value. Hence, the objective function in (14) is increasing over $n \in\left[0, \tilde{n}^{*}\right)$ and decreasing over $n \in\left[\tilde{n}^{*}, \infty\right)$, where $\tilde{n}^{*}=2 B / \tilde{P}^{*}$. Hence, with an integer constraint on $n$, the objective function in (13) attains the maximum value at either or both of the following values of $n: n=\left\lfloor\tilde{n}^{*}\right\rfloor$ and $n=\left\lceil\tilde{n}^{*}\right\rceil$, where $\lfloor x\rfloor$ is the greatest integer smaller than $x$ and $\lceil x\rceil$ is the smallest integer greater than $x$. Therefore, the optimal $n$ that solves (13) is given by

$$
n^{*}=\underset{n \in\left\{\left\lfloor\tilde{n}^{*}\right\rfloor,\left\lceil\tilde{n}^{*}\right\rceil\right\}}{\arg \max } \frac{\frac{n}{2} \log \left(1+\frac{2 B}{n}\right)}{n+\frac{2 B}{\mu}},
$$

and the maximum long-term average throughput is given by

$$
T_{\mathrm{SB}}=\frac{\frac{n^{*}}{2} \log \left(1+\frac{2 B}{n^{*}}\right)}{n^{*}+\frac{2 B}{\mu}} .
$$

We now make the following remarks.

- From Theorem 2, which gives the optimal solution to the relaxed long-term average throughput maximization problem when $B \geq E_{H}$, we note that the optimal power and throughput depend only on $\mu$, i.e., for any $B$ such that $B \geq E_{H}$, the optimal power and throughput remain the same. In other words, the performance with an infinitecapacity battery can be obtained by using a single battery with capacity of $2 B=2 E_{H}$ units, in the relaxed case.

- In the presence of integer constraints, from (17) and (18), the optimal performance depends only on the mean value of the harvested energy and the battery capacity. This implies that burstiness of the energy arrivals does not impact the optimal performance.

- In order to obtain Lemma 1, we have not used the fact that energy arrivals admit the Bernoulli EH model in (1). The term $\bar{L}_{2 B}$ is simply the expected value of the number of slots required to accumulate at least $2 B$ units of energy in an arbitrary i.i.d. EH model (see (11)). Moreover, we can extend the optimization problem in (14) to an arbitrary i.i.d. EH model by replacing $\mu$ by $2 B / \bar{L}_{2 B}$. This implies that, Lemma 1, Theorem 2, (17) and (18) are readily applicable to an arbitrary i.i.d. EH model when $\mu$ is replaced by $2 B / \bar{L}_{2 B}$.

\section{P2P Channel: DUAL BATtery CASE}

We now consider the P2P channel under the dual battery case. In the sequel, we solve (10) in the online case via dynamic programming, in which the optimal transmit power in a slot is obtained based on the state of the batteries in the slot. We then propose non-adaptive online policies, which do not need knowledge of the current state. Based on this, we also propose simple, easy to implement policies which adapt the power allocation whenever a new energy arrival occurs. We begin with the following lemma. 
Lemma 3: It is optimal to switch the roles of the batteries when the working battery becomes empty and the charging battery becomes full.

Proof: See Appendix C.

The above lemma has the following two implications.

- First, due to Lemma 3, the switching instants are renewal instants of the battery state processes. Let $C_{k}$ denote the length (in slots) of the $k^{\text {th }}$ renewal period. Since the system is reset after a renewal instant and because the energy arrivals and allocations are independent across renewal intervals, $C_{1}, C_{2}, \ldots$ form a sequence of i.i.d. random variables. Hence, by the renewal-reward theorem [27] and (8), the long-term average throughput is

$$
T=\frac{\mathbb{E}\left[\sum_{i=1}^{C} \frac{1}{2} \log \left(1+P_{i}\right)\right]}{\mathbb{E}[C]},
$$

where $C$ is the length of the renewal period, whose distribution is identical to $C_{1}, C_{2}, \ldots$ The expectation is with respect to the random variable $C$.

- Second, due to Lemma 3, at any point in time, only one of the batteries will be the charging battery and the other will be the working battery and hence, we have, $\alpha_{1, i}=$ $1-\alpha_{2, i}$ and $\beta_{j, i}=\alpha_{j, i}$ for $j \in\{1,2\}$ in (5) and (6). Therefore, we can describe the roles and evolution of the batteries in (5) and (6) with a single indicator variable, denoted by $\alpha$, as follows. For $i \in\{1,2, \ldots\}$,

$$
\begin{aligned}
B_{1, i+1} & =\max \left(\min \left(B_{1, i}+\left(1-\alpha_{i}\right) E_{i}, B\right)-\alpha_{i} P_{i}, 0\right), \\
B_{2, i+1} & =\max \left(\min \left(B_{j, i}+\alpha_{i} E_{i}, B\right)-\left(1-\alpha_{i}\right) P_{i}, 0\right), \\
\alpha_{i+1} & = \begin{cases}0 & \mathbb{1}_{\{0\}}\left(B_{1, i+1}\right) \mathbb{1}_{\{B\}}\left(B_{2, i+1}\right), \\
1 & \mathbb{1}_{\{B\}}\left(B_{1, i+1}\right) \mathbb{1}_{\{0\}}\left(B_{2, i+1}\right), \\
\alpha_{i} & \text { otherwise, }\end{cases}
\end{aligned}
$$

where $\alpha_{i}=1\left(\alpha_{i}=0\right)$ indicates that, in slot $i$, the second battery is the charging (working) battery and the first battery is the working (charging) battery.

Before we proceed, we define certain quantities. The number of slots required to accumulate $B$ units of energy, $L$, follows the negative binomial distribution given by

$$
\operatorname{Prob}(L=m) \triangleq q_{m}=\left(\begin{array}{c}
m-1 \\
m-r
\end{array}\right) p^{r}(1-p)^{m-r},
$$

for $m \in\{r, r+1, \ldots\}$ and $\mathbb{E}[L]=r / p$. Further, the cumulative density function (CDF) is given by, $F_{i}(r, p)=\sum_{m=1}^{i} q_{m}$ and the complementary $\mathrm{CDF}, \bar{F}_{i}(r, p)=1-F_{i}(r, p)=$ $\sum_{m=i+1}^{\infty} q_{m}$. In the following subsection, we present several online policies. For completeness of benchmarking, we also present the optimal offline policy in Appendix D.

\section{A. Optimal Online Policy}

We now consider the online case with only causal knowledge of energy arrivals. Since the precise time when the charging battery will get full is unknown, power is allocated based only on the distribution of energy arrivals. To obtain the optimal online policy, we adopt a dynamic programming framework. We now define the relevant quantities.
1) State Space: The state of the system is defined by 3-tuples $s \triangleq\left(b_{1}, b_{2}, \alpha\right)$, where $b_{1}$ and $b_{2}$ are the amounts of energy stored in the first and the second battery, respectively, at the start of a slot and $\alpha$ is an indicator variable, where $\alpha=1(\alpha=0)$ indicates that the second battery is the charging (working) battery and the first battery is the working (charging) battery. The state space

$$
\mathcal{S}=\left\{\left(b_{1}, b_{2}, \alpha\right): 0 \leq b_{1}, b_{2} \leq B, \alpha \in\{0,1\}\right\} .
$$

2) Action Space and Reward: The action space the system can take in state $s=\left(b_{1}, b_{2}, \alpha\right) \in \mathcal{S}$,

$$
\mathcal{A}(s)=\left\{P: 0 \leq P \leq b_{1} \alpha+b_{2}(1-\alpha)\right\} .
$$

The constraint on $P$ in (23) is due to the fact that $P$ units are drawn from the working battery.

3) State Transition Probability Matrix: The state transition is fully specified by (20)-(22), based on which, we can easily construct the probability transition matrix, $q\left(s^{\prime} \mid P, s\right)$ for all $s^{\prime}, s \in \mathcal{S}$ and $P \in \mathcal{A}(s)$.

4) Optimal Value Function: We consider $K$ slots for the optimization. We obtain the optimal value function $V_{k}(s)$ in slot $k \in\{1, \ldots, K\}$ and state $s \in \mathcal{S}$ by solving the following Bellman equation:

$$
V_{k}(s)=\max _{P \in \mathcal{A}(s)}\left(\frac{1}{2} \log (1+P)+\sum_{s^{\prime} \in \mathcal{S}} q\left(s^{\prime} \mid P, s\right) V_{k+1}\left(s^{\prime}\right)\right),
$$

for $k=K, K-1, \ldots, 1$, where $V_{K+1}(s) \triangleq 0$. The optimal online throughput is then given by

$$
T_{\text {on }}=\liminf _{K \rightarrow \infty} \frac{V_{K}(s)}{K} .
$$

The optimal online throughput, $T_{\text {on }}$, can also be obtained by solving the Bellman equation in the infinite horizon case with the discount factor arbitrarily close to one. Since the reward is bounded and the state space is finite, there exists an optimal stationary deterministic policy for (24), i.e., there exists a unique optimal action $P^{*}(s)$ in state $s$ independent of slot indices [29]. Hence, it suffices to search only in the set of all stationary deterministic policies.

The dynamic programming framework can be applied for an arbitrary i.i.d. energy arrival model to obtain an optimal online policy. However, this requires one to adopt a more general state and action spaces, based on (5)-(7). Note that the state and action spaces used in the above dynamic programming based policy is based on Lemma 3, which is specific to the Bernoulli EH model.

\section{B. Non-Adaptive (NA) Online Policies}

The above optimal online policy, obtained via dynamic programming, adapts the transmit power in every slot based on the state of the batteries. In the non-adaptive policies, we assume the states of batteries are not estimated in every slot. However, we assume that a flag is raised when the charging battery becomes full or working battery becomes empty. Further, the energy remaining in the working battery is discarded once the charging battery accumulates $B$ units of energy. On the other 
hand, if the working battery gets completely discharged before the charging battery is full, the transmitter waits without transmission till the charging battery gets full. This implies that the renewal instant is the same as the instant when the charging battery accumulates $B$ units of energy. With this setting, we obtain optimal and suboptimal power allocations in the following. For our analysis, we adopt the approach developed in [14].

1) Optimal Non-Adaptive (ONA) Online Policy: Let $\tilde{P}_{i}$ be the transmit power in slot $i \in\{1, \ldots, L\}$ after a renewal. Then, the throughput in (19) can be re-written as

$$
T_{\mathrm{ONA}}=\sum_{i=1}^{\infty} \frac{p}{2 r} \bar{F}_{i-1}(r, p) \log \left(1+\tilde{P}_{i}\right)
$$

where we recall $\bar{F}_{i-1}(r, p)=\sum_{m=i}^{\infty} q_{m}$. From (10) and (25), to maximize the long-term average throughput in the online case, we need to solve the following optimization problem:

$$
\begin{aligned}
& \underset{\tilde{P}_{i}, i=1,2, \ldots}{\operatorname{maximize}} \sum_{i=1}^{\infty} \frac{p}{2 r} \bar{F}_{i-1}(r, p) \log \left(1+\tilde{P}_{i}\right), \\
& \text { subject to } \sum_{i=1}^{\infty} \tilde{P}_{i} \leq B, \quad \tilde{P}_{i} \geq 0, i \in\{1,2, \ldots\} .
\end{aligned}
$$

Clearly, (26) is a convex optimization problem. Hence, the Karush-Kuhn-Tucker (KKT) conditions are necessary and sufficient for optimality. Based on the KKT conditions, we obtain the optimal power allocations in the following theorem.

Theorem 4: The optimal transmit power in the $i^{\text {th }}$ slot after a renewal instant

$$
\tilde{P}_{i}^{\text {ONA }}(B, r, p)= \begin{cases}\frac{(B+M)}{\sum_{j=1}^{M} \bar{F}_{j-1}(r, p)}-1, & \forall i=1, \ldots, r, \\ \frac{(B+M) \bar{F}_{i-1}(r, p)}{\sum_{j=1}^{M} \bar{F}_{j-1}(r, p)}-1, & \forall i=r+1, \ldots, M, \\ 0, & \forall i>M,\end{cases}
$$

where $M \triangleq \max \left\{m: \frac{\sum_{i=1}^{m} \bar{F}_{i-1}(r, p)}{B+m} \leq \bar{F}_{m-1}(r, p)\right\}$. The corresponding throughput in the ONA policy can be obtained from (25) and (27) as

$$
T_{\mathrm{ONA}}=\sum_{i=1}^{M} \frac{p}{2 r} \bar{F}_{i-1}(r, p) \log \left(\frac{(B+M) \bar{F}_{i-1}(r, p)}{\sum_{j=1}^{M} \bar{F}_{j-1}(r, p)}\right) .
$$

Proof: See Appendix E.

We note that the probability that the charging battery fills up in less than $r$ slots after a renewal is zero. Hence, given a fixed amount of energy that can be consumed in the first $r$ slots, it is intuitive that we must consume it at a constant rate in the first $r$ slots after a renewal, as suggested by (27). We also note that under the policy $\tilde{P}_{\text {ONA }}$, the working battery becomes fully discharged after exactly $M$ slots. It strikes the optimal balance between discharging too early (in which case the transmitter will remain idle till the charging battery gets full) and discharging too late (in which case there is wastage of energy as the remaining energy in the working battery is discarded). Since we discard the remaining energy in the working battery in case the charging battery becomes full before $M$ slots and we remain idle in case the charging battery takes

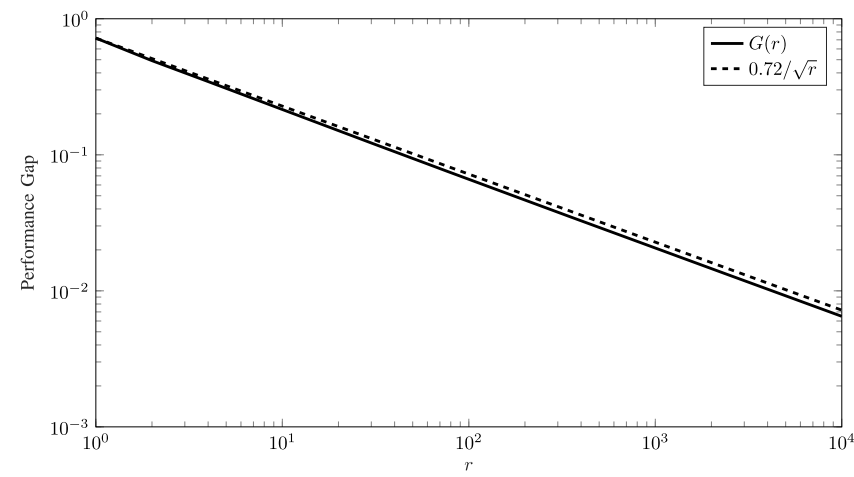

Fig. 2. Variation of $G(r)$ with $r$.

more than $M$ slots to become full, the solution in Theorem 4 is a suboptimal online policy and $T_{\mathrm{ONA}} \leq T_{\mathrm{ub}}$.

2) Suboptimal Non-Adaptive (SNA) Online Policy: Based on the above ONA policy, we now propose a suboptimal policy, referred to as the Suboptimal Non-Adaptive (SNA) policy. Our motivations for proposing the SNA policy are it is simpler than the previous policies, and analytically tractable in the sense that we can use it to lower bound the optimal long-term average throughput in the dual battery case.

In the SNA policy, the power allocation in the $i^{\text {th }}$ slot after a renewal instant is given by

$$
\tilde{P}_{i}^{\mathrm{SNA}}(B, r, p)=\frac{B p}{r} \sum_{m=i}^{\infty} q_{m}=\mu \bar{F}_{i-1}(r, p) .
$$

We discard the energy remaining in the working battery when the charging battery becomes full. Since $\sum_{i=1}^{\infty} \bar{F}_{i-1}(r, p)=$ $\sum_{i=1}^{\infty} \sum_{m=i}^{\infty} q_{m}=\mathbb{E}[L]=r / p$, we note, $\sum_{i=1}^{\infty} \tilde{P}_{i}^{\mathrm{SNA}}=B$. Hence, the power allocation policy in (28) does not violate the energy causality constraint. It is also interesting to note that when $r=1$, we recover the FFP proposed in [14]. Let $T_{\text {SNA }}$ denote the long-term average throughput obtained in this strategy. We now have the following result.

Theorem 5: The long-term average throughput in the SNA policy is bounded as follows:

$$
T_{\mathrm{ub}} \geq T_{\mathrm{SNA}} \geq T_{\mathrm{ub}}-G(r),
$$

where $G(r) \triangleq \max _{p}-\frac{p}{2 r} \sum_{i=1}^{\infty}\left(\sum_{m=i}^{\infty} q_{m}\right) \log \left(\sum_{m=i}^{\infty} q_{m}\right)$ and $q_{m}=\left(\begin{array}{c}m-1 \\ m-r\end{array}\right) p^{r}(1-p)^{m-r}$.

Proof: See Appendix F.

We make the following remarks on the above theorem.

- First, $G(1) \approx 0.72$ and we recover [14, Proposition 3].

- Further, numerically, we can show that $\max _{r} G(r)=$ $G(1) \approx 0.72$. Hence, the long-term average throughputs in the ONA and the SNA policies are at most 0.72 bits away from the upper bound $T_{\mathrm{ub}}$ for any value of $p, B$ and $r$, under the Bernoulli energy harvesting model.

- Finally, when the parameters $E_{H}$ and $p$ are fixed, $r$ increases proportionately with $B$, as $r=B / E_{H}$. From numerical analysis, we find the bound $G(r)$ decreases monotonically at a rate faster than the inverse of the square root of $r$ and $B$, as shown in Fig. 2 . 


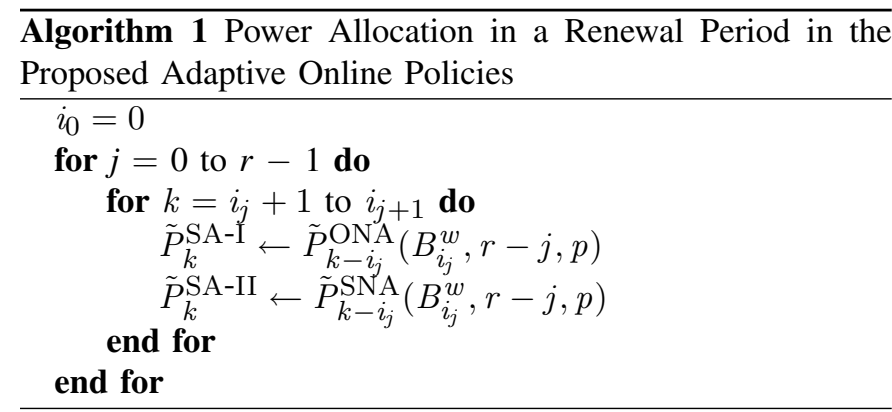

\section{Adaptive Online Policies}

Here, we assume the state of the charging and working batteries, denoted by $B^{c}$ and $B^{w}$, respectively, are known at the start of every slot. In this policy, we adapt the power allocations based on $B^{c}$ and $B^{w}$. Recall that, by assumption, it takes $r$ energy arrivals to fill the charging battery, starting from the empty state. Now, until the current slot, suppose that $j$ energy arrivals have occurred since the last renewal instant, i.e., $B^{c}=j E_{H}$. Then, we need $r-j$ more energy arrivals to fill the battery. Let $L_{r-j}$ be the random number of slots required for $r-j$ energy arrivals. Then, the complementary CDF of $L_{r-j}$ is given by $\bar{F}_{i}(r-j, p), i \in\{1,2, \ldots\}$, where we recall $\bar{F}_{i}(r, p)=\sum_{m=i+1}^{\infty} q_{m}$. Now, in the adaptive policy, we target to allocate $B^{w}$ units of energy in the working battery based on the distribution of $L_{r-j}$, along the lines in the non-adaptive policies proposed earlier. Concretely, the power allocation to $i^{\text {th }}$ slot after $j^{\text {th }}$ energy arrival in a renewal window is given by $\tilde{P}_{i}^{\mathrm{ONA}}\left(B^{w}, r-j, p\right)$, where $\tilde{P}_{i}^{\mathrm{ONA}}(\cdot)$ is defined in (27). We refer to this policy as the SuboptimalAdaptive I (SA-I) policy. Similarly, we obtain the policy, which we refer to as the Suboptimal-Adaptive II (SA-II) policy, by allocating $\tilde{P}_{i}^{\mathrm{SNA}}\left(B^{w}, r-j, p\right)$ units of energy to $i^{\text {th }}$ slot after $j^{\text {th }}$ energy arrival in a renewal window, where $\tilde{P}_{i}^{\mathrm{SNA}}(\cdot)$ is defined in (28). The policies SA-I and SA-II may be suboptimal, hence we refer to them as Suboptimal-Adaptive policies (notice that the dynamic programming based policy is the optimal adaptive online policy). We summarize the power allocations in these adaptive policies in Algorithm 1. In the algorithm, $i_{j}$ represents the index of $j^{\text {th }}$ energy arrival in a renewal window. Further, we represent the long-term average throughputs obtained by SA-I and SA-II policies by $T_{\mathrm{SA}-\mathrm{I}}$ and $T_{\mathrm{SA}-\mathrm{II}}$, respectively.

\section{Constant Power (CP) Policy}

In the $\mathrm{CP}$ policy, the transmit power remains constant whenever transmission occurs. Our motivations for proposing this policy are the following. First, practical implementation of such a policy is simple as it does not require the knowledge of battery states. Further, prior knowledge of the optimal transmit power can enable system designers to choose appropriate system components such as power amplifiers such that optimal transmit power is in their linear operating region. Finally, several variants of this policy, considered in [14] and references therein, are shown to perform competitively with optimal policies. Specifically, the version proposed in [25] has been shown to approach $T_{\mathrm{ub}}$ asymptotically with $B$. Hence, this policy is also useful in benchmarking the policies studied above. In the $\mathrm{CP}$ policy, we consume the energy available in the battery at a constant rate of $B /\left(\left\lfloor r p^{-1}\right\rfloor\right)$ as long as the battery is not empty, i.e., the power allocation in the $i^{\text {th }}$ slot after a renewal instant is given by

$$
\tilde{P}_{i}=\frac{B}{\left\lfloor r p^{-1}\right\rfloor}, \quad \forall i=1, \ldots,\left\lfloor r p^{-1}\right\rfloor .
$$

We discard the remaining energy in the working battery when the charging battery becomes full. We denote the long-term average throughput of the policy by $T_{\mathrm{CP}}$.

\section{MAC: Single Battery And Dual BatTery Cases}

We now consider a $U$-user MAC where the users communicate to a common receiver over an AWGN channel with unit noise power. Let $\mathcal{U} \triangleq\{1, \ldots, U\}$ represent the set of user indices. In the single battery case, we assume that user $u \in \mathcal{U}$ is equipped with a single battery of capacity $2 B_{u}$ units. Similarly, in the dual battery case, we assume that user $u \in \mathcal{U}$ is equipped with two identical batteries, each having the capacity of $B_{u}$ units. We apply the half-duplex battery constraint and the cycle constraint described in Section II. The energy arrivals are i.i.d. over time and the amount of energy harvested in slot $i$ by user $u$ is

$$
E_{u i}= \begin{cases}E_{H_{u}} & \text { w.p. } p_{u} \\ 0 & \text { w.p. } 1-p_{u}\end{cases}
$$

We assume that $B_{u} / E_{H_{u}}=r_{u}$ for some $r_{u} \in\{1,2, \ldots\}$ and we note the average $\mathrm{EH}$ rate, $\mu_{u}=p_{u} E_{H_{u}}$ at user $u \in \mathcal{U}$. The battery evolution at each user is similar to that in the P2P channel case, described in Section II-B. Let the transmit power of user $u$ in slot $i$ be denoted by $P_{u i}$. Then, from [15], the maximum average throughput region, averaged over all the sample paths of energy arrivals is given by

$$
\begin{aligned}
\mathcal{T}_{K}\left(P_{u k}\right)=\left\{R_{u}: \sum_{u \in \mathcal{S}} R_{u}\right. \\
\left.\quad \leq \frac{1}{K} \mathbb{E}\left[\sum_{k=1}^{K} \frac{1}{2} \log \left(1+\sum_{u \in \mathcal{S}} P_{u k}\right)\right], \forall \mathcal{S} \subseteq \mathcal{U}\right\} .
\end{aligned}
$$

Our goal is to maximize the long-term average throughput region defined as $\mathcal{T}=\liminf _{K \rightarrow \infty} \mathcal{T}_{K}$. We now present an outer bound to $\mathcal{T}$ in the following. When the cycle and halfduplex battery constraints are not present, and the capacities of the batteries are infinite, the largest throughput region for the Gaussian MAC is given by

$$
\mathcal{T}_{\text {outer }}=\left\{R_{u}: \sum_{u \in \mathcal{S}} R_{u} \leq \frac{1}{2} \log \left(1+\sum_{u \in \mathcal{S}} \mu_{u}\right), \forall \mathcal{S} \subseteq \mathcal{U}\right\}
$$

where $\mu_{u}$ is the mean energy arrival rate in user $u \in \mathcal{U}$ [15].

In the sequel, we propose achievable strategies in the single battery and the dual battery cases, based on the P2P channel studied in the previous sections. 


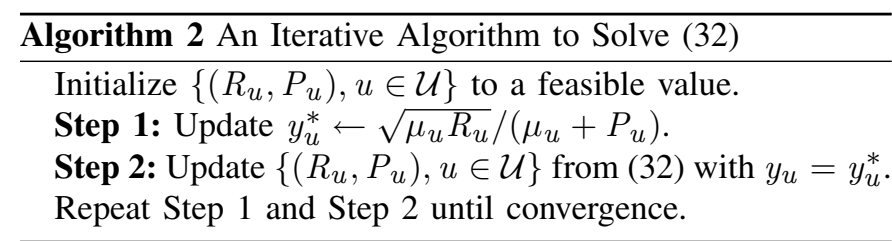

\section{A. Single Battery Case}

Here, as in the P2P channel case studied in Section III, we assume that each user transmits with a constant power. We only consider the relaxed problem for simplicity. Let $\left(R_{u}, P_{u}\right)$ be transmit rate and power pairs for user $u \in \mathcal{U}$. From (14), the long-term average throughput of user $u$ is given by $T_{u}=$ $\mu_{u} R_{u} /\left(\mu_{u}+P_{u}\right)$. We now note that each boundary point on the largest achievable throughput region is the optimal solution

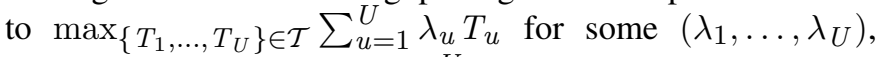
where $\lambda_{u} \geq 0, u \in \mathcal{U}$ and $\sum_{u=1}^{U} \lambda_{u}=1$. Hence, we obtain all the boundary points on the achievable throughput region in this policy by solving the following optimization problem for many different instances of $\left(\lambda_{1}, \ldots, \lambda_{U}\right)$ :

$$
\begin{aligned}
\underset{R_{u}, P_{u} \geq 0}{\operatorname{maximize}} & \sum_{u=1}^{U} \frac{\lambda_{u} \mu_{u} R_{u}}{\mu_{u}+P_{u}}, \\
\text { subject to } & \sum_{u \in \mathcal{S}} R_{u} \leq \frac{1}{2} \log \left(1+\sum_{u \in \mathcal{S}} P_{u}\right),
\end{aligned}
$$

for all $u \in \mathcal{U}$ and $\mathcal{S} \subseteq \mathcal{U}$. The above optimization problem is non-convex as (31a) is a sum of ratios. Hence, we transform (31) into the following equivalent optimization problem:

$$
\begin{aligned}
& \underset{\substack{R_{u}, P_{u} \geq 0 \\
y_{u} \in \mathbb{R}}}{\operatorname{maximize}}, \sum_{u=1}^{U} \lambda_{u}\left(2 y_{u} \sqrt{\mu_{u} R_{u}}-y_{u}^{2}\left(\mu_{u}+P_{u}\right)\right), \\
& \text { subject to } \\
& \sum_{u \in \mathcal{S}} R_{u} \leq \frac{1}{2} \log \left(1+\sum_{u \in \mathcal{S}} P_{u}\right),
\end{aligned}
$$

for all $u \in \mathcal{U}$ and $\mathcal{S} \subseteq \mathcal{U}$. The equivalence of (31) and (32) follows from [30, Corollary 1]. The above problem can be solved by alternate maximization over $\left\{y_{u}, u \in \mathcal{U}\right\}$ and $\left\{\left(R_{u}, P_{u}\right), u \in \mathcal{U}\right\}$, as shown in Algorithm 2. Step 1 in Algorithm 2 is because, for a fixed $\left\{\left(R_{u}, P_{u}\right), u \in \mathcal{U}\right\}$, the optimal $y_{u}^{*}$ in (32) is given by $\sqrt{\lambda_{u} \mu_{u} R_{u}} /\left(\mu_{u}+P_{u}\right)$. The convergence of the above maximization problem to a stationary point follows from [30, Th. 3].

\section{B. Dual Battery Case}

We now consider the dual battery case and present the following result on the inner region. To obtain the inner region, we assume each user adopts the SNA policy in (28) individually.

Proposition 6: The long-term average throughput region, $\mathcal{T}_{\text {inner_DB }} \subseteq \mathcal{T}$, where, $\mathcal{T}_{\text {inner_DB }}=\left\{R_{u}: \sum_{u \in \mathcal{S}} R_{u} \leq\right.$ $\left.\frac{1}{2} \log \left(1+\sum_{u \in \mathcal{S}} \mu_{u}\right)-\max \left\{G\left(r_{1}\right), \ldots, G\left(r_{U}\right)\right\}, \forall \mathcal{S} \subseteq \mathcal{U}\right\}$

Proof: The result can be obtained by extending Theorem 5 along the lines of the proof of $[15$, Th. 1].

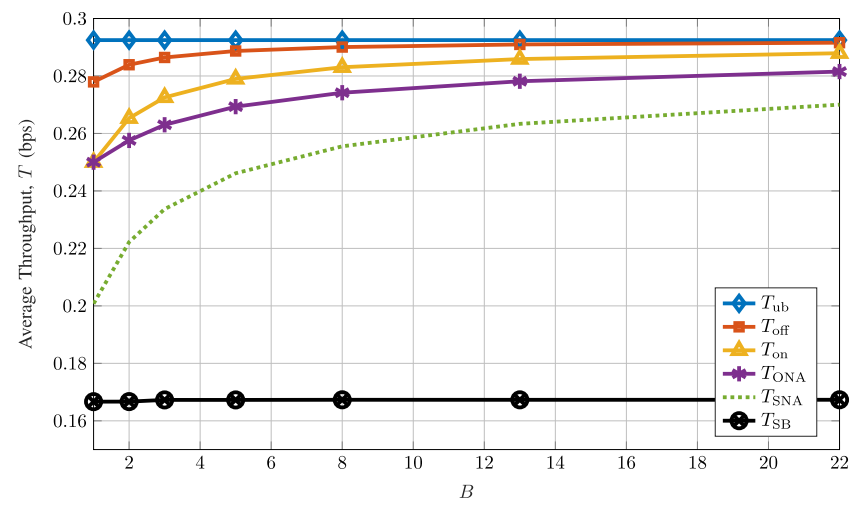

Fig. 3. Variation of the optimal long-term average throughput with $B$ for $p=0.1, E_{H}=1$ and with $r=B$.

We now propose an achievable scheme based on the ONA policy described in the P2P channel case. Let $R_{u i}$ and $P_{u i}$ be the transmit rate and power in slot $i$ of user $u$. Then, from (25), we recall that the long-term average throughput in user $u$ is given by $\sum_{i=1}^{\infty}\left(p_{u} / r_{u}\right) \bar{F}_{u(i-1)} R_{u i}$, where $\bar{F}_{u i}=1-F_{u i}=$ $\sum_{m=i+1}^{\infty} q_{u m}$ and $q_{u m} \triangleq\left(\begin{array}{c}m-1 \\ m-r_{u}\end{array}\right) p_{u}^{r_{u}}\left(1-p_{u}\right)^{m-r_{u}}$. Hence, along the lines in the previous subsection, we obtain all the boundary points on the largest achievable throughput region by solving the following convex optimization problem for different instances of $\left(\lambda_{1}, \ldots, \lambda_{U}\right)$ with $\sum_{u=1}^{U} \lambda_{u}=1$ :

$$
\begin{aligned}
\underset{R_{u i}, P_{u i}}{\operatorname{maximize}} & \sum_{i=1}^{\infty} \sum_{u=1}^{U} \frac{\lambda_{u} p_{u}}{r_{u}} \bar{F}_{u(i-1)} R_{u i}, \\
\text { subject to } & \sum_{i=1}^{\infty} P_{u i} \leq B_{u}, R_{u i}, P_{u i} \geq 0, \\
& \sum_{u \in \mathcal{S}} R_{u i} \leq \frac{1}{2} \log \left(1+\sum_{u \in \mathcal{S}} P_{u i}\right),
\end{aligned}
$$

for all $u \in \mathcal{U}, \mathcal{S} \subseteq \mathcal{U}$ and $i \in\{1,2, \ldots\}$. The above problem is convex, and hence, it can be solved efficiently using standard numerical techniques. In this policy, we note that user $u$ transmits with power $P_{u i}$ and rate $R_{u i}$ in slot $i$ after a renewal, independent of the rates and transmit powers of the other users.

\section{Numerical Results}

In this section, we first compare the long-term average throughputs obtained in the P2P channel under the single battery and dual battery cases. We then obtain long-term average throughput regions in a MAC using the schemes presented in the previous section. The parameters used for our simulations are in the similar range as in [14], [16], [17].

\section{A. Long-Term Average Throughput in a P2P Channel}

In Fig. 3, we plot variation of the long-term average throughput with the battery capacity in various policies. We note that as the battery capacity increases, the performance gap between offline (see Appendix D) and online policies decreases. Further, the average throughput achieved by the ONA policy approaches the upper bound, $T_{\mathrm{ub}}$, 


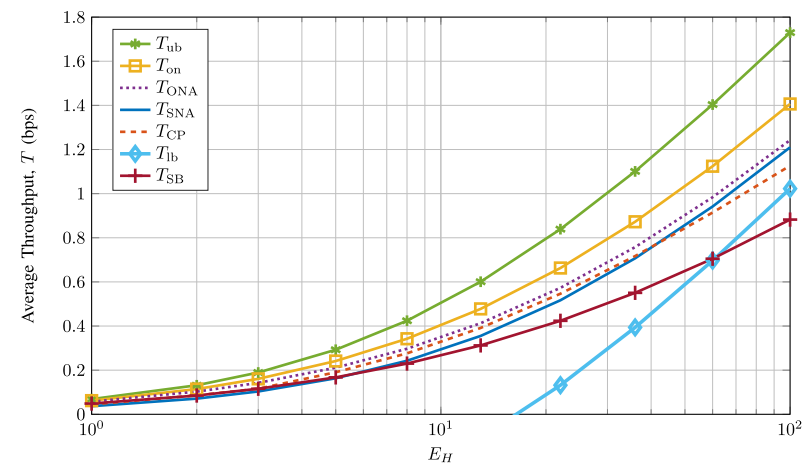

(a) When $B=E_{H}$.

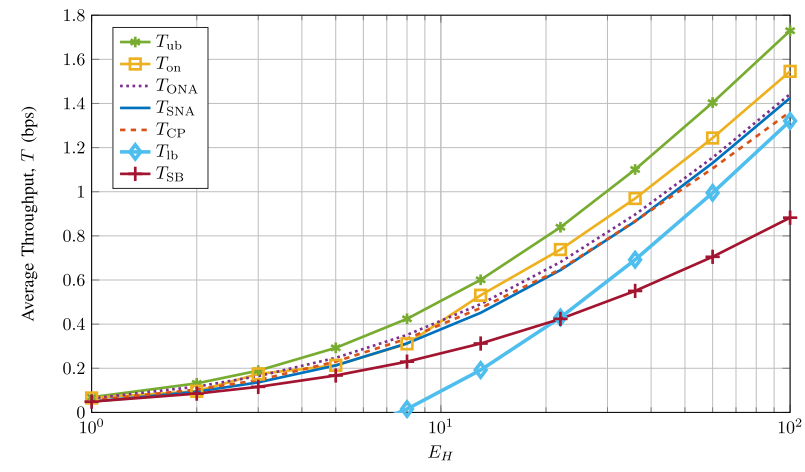

(b) When $B=3 E_{H}$.

Fig. 4. Variation of the optimal long-term average throughput with $E_{H}$ when $B=E_{H} r$ for $r=1,3$ and $p=0.1$.

much faster than the SNA policy, as the battery capacity $B$ increases. The long-term average throughput in the single battery case, $T_{\mathrm{SB}}$, does not depend on the battery capacity. This is because, as seen in (16), the maximum long-term average throughput for the relaxed problem in (14) depends only on the average harvested power; rounding the solution introduces a negligible change in the throughput of the relaxed problem.

In Fig. 4, we plot variation of the long-term average throughput with the amount of energy harvested per arrival, $E_{H}$, for $B=E_{H}$ (see Fig. 4a) and $B=3 E_{H}$ (see Fig. 4b). When $B=E_{H}$, a single energy arrival completely fills up the battery. In this scenario, the system model of the current paper in the dual battery case is identical to that in [14], and Fig. 4a is similar to [14, Fig. 4], where ONA and SNA policies of the current paper correspond to the optimal policy and the constant-fraction policy of [14], respectively. In this case, the long-term average throughputs in ONA and SNA policies are at most 0.72 bits away from the upper bound, as pointed out in the remarks on Theorem 4. In Fig. 4b, we set $B=3 E_{H}$. For a given $E_{H}$, note that the mean value of the harvested energy, $\mu=p E_{H}$, is the same in both the figures. Hence, the upper bound remains the same in both the figures. However, the performance of the optimal offline, optimal online, ONA and SNA policies when $B=3 E_{H}$ are better than that when $B=E_{H}$. Based on Theorem 4, the long-term average throughputs in ONA and SNA policies are at most 0.41 bits away from the upper bound when $B=3 E_{H}$, as illustrated by the $T_{\mathrm{lb}} \triangleq T_{\mathrm{ub}}-G(r)$ curve. From Fig. 4a,
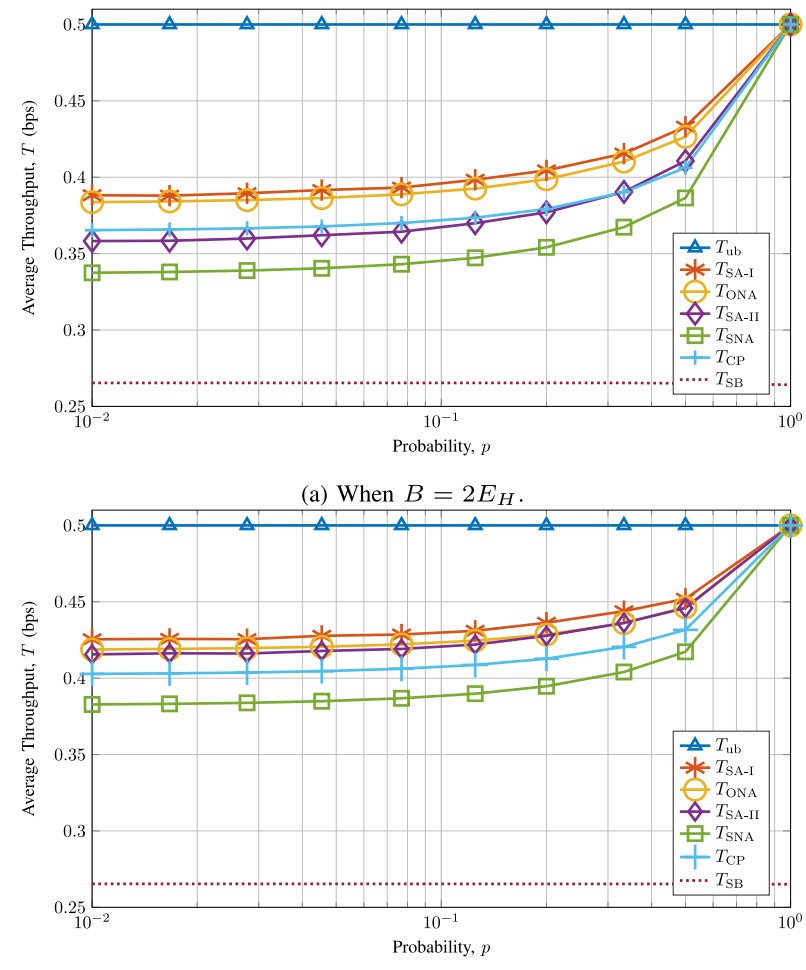

(b) When $B=4 E_{H}$

Fig. 5. Variation of the optimal long-term average throughput with $p$ for a fixed mean harvesting rate, $\mu=1$, with $B=r E_{H}$ for $r=2,4$.

we also note that the $\mathrm{CP}$ policy performs better than the SNA policy when $E_{H}$ is small. However, as $E_{H}$ increases, the performance gap of the $\mathrm{CP}$ policy from the upper bound increases, unlike the SNA and ONA policies, which maintain a bounded gap from the upper bound. This observation has been made for $B=E_{H}$ case in [14]. A similar observation holds when $B=3 E_{H}$. In the single battery case, the performance curves are almost identical in both the figures. This shows that, as highlighted in Section III, increasing $B$ has a negligible impact on the performance, provided $B \geq E_{H}$. We also note that the performance gap of the single battery case from the upper bound diverges as $E_{H}$ increases. Finally, since the performance of the optimal online, ONA and SNA policies of the dual battery case are within a constant gap from the upper bound, and as the performance of the single battery case diverges with $E_{H}$, we conclude that the performance of the dual battery case can be more than twice of that in the single battery case for high $E_{H}$.

In Fig. 5, we plot variation of the long-term average throughput with $p$, for a fixed mean harvesting rate of $\mu=1$, for $B=2 E_{H}$ (see Fig. 5a) and $B=4 E_{H}$ (see Fig. 5b). For the same parameters, we also present the average idle time, the fraction of the slot length over which the transmit power is zero, in Table I, and we plot the variation of the average amount of energy discarded per slot, $E_{\text {discarded, with } p \text { in }}$ Fig. 6. Note that the lower the value of $p$, the higher is the amount of energy harvested per arrival, i.e., the energy arrival becomes more bursty as $p$ is decreased keeping $\mu$ fixed.

We make the following key observations from Fig. 5, Table I and Fig. 6. Firstly, the long-term average throughputs in all the 
TABLE I

AVerage Idle Time, the Fraction of the Slot Length Over Which THE TRANSMIT POWER IS ZERo (EXPRESSED AS THE Percentage of the Slot Length), FOR $\mu=1$

\begin{tabular}{|l|c|l|l|l|}
\hline Policies & \multicolumn{3}{|c|}{ Average idle time (\% of the slot length) } \\
\hline & \multicolumn{2}{|c|}{$B=2 E_{H}$} & \multicolumn{2}{c|}{$B=4 E_{H}$} \\
\cline { 2 - 5 } & $p=0.01$ & $p=0.5$ & $p=0.01$ & $p=0.5$ \\
\hline SA-I Policy & $31.0 \%$ & $22.0 \%$ & $26.0 \%$ & $15.0 \%$ \\
\hline ONA Policy & $26.0 \%$ & $19.0 \%$ & $20.0 \%$ & $12.0 \%$ \\
\hline SA-II Policy & $0.62 \%$ & $0.59 \%$ & $0.3 \%$ & $0.2 \%$ \\
\hline SNA Policy & $0.2 \%$ & $0.15 \%$ & $0.17 \%$ & $0.05 \%$ \\
\hline CP Policy & $29.0 \%$ & $19.0 \%$ & $22.0 \%$ & $12.0 \%$ \\
\hline SB Optimal Policy & $63.0 \%$ & $62.0 \%$ & $63.0 \%$ & $63.0 \%$ \\
\hline
\end{tabular}

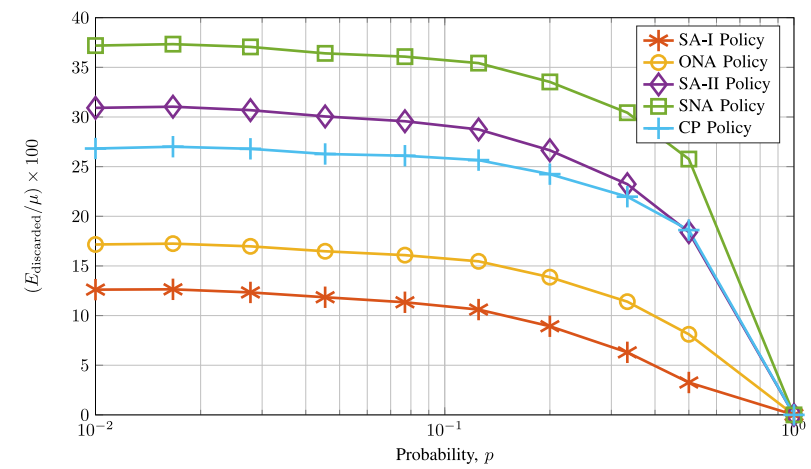

(a) When $B=2 E_{H}$.

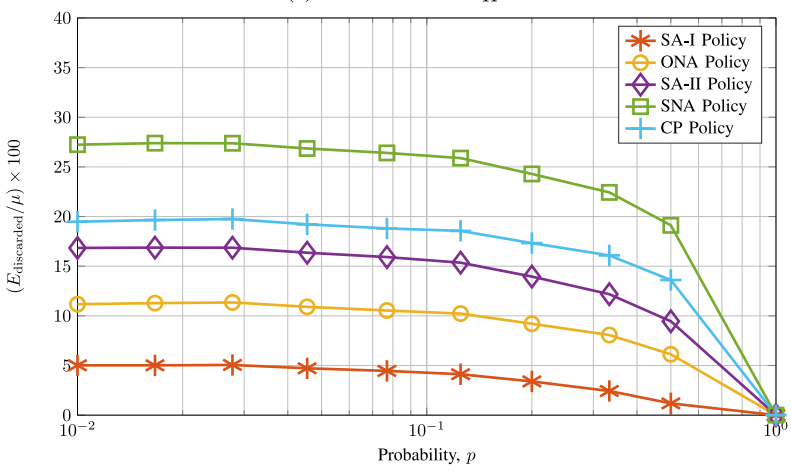

(b) When $B=4 E_{H}$.

Fig. 6. Variation of the average amount of energy discarded per slot, $E_{\text {discarded }}$ (expressed as the percentage of the mean energy arrival rate, $\mu$ ), with $p$ for a fixed mean harvesting rate $\mu=1$, with $B=r E_{H}$ for $r=2,4$.

policies are significantly lower than the upper bound when $p$ is small and they approach the upper bound as $p$ is increased. This is because, as seen from Table I, when $p$ is small, the average idle time is large. This indicates that, when $p$ is small, all the policies are aggressive, i.e., $B$ units of energy in the working battery is consumed in a smaller duration of time compared to the case when $p$ is higher. Due to the concavity of the throughput, this leads to a degradation in the throughput. Further, as seen from Fig. 6, the average amount of energy discarded when $p$ is small is more than that when $p$ is larger. This also reduces the achievable throughput. For the higher values of $p$, the harvested energy arrives more uniformly and the power allocation can be nearly constant, and the average idle time and the average amount of energy discarded decrease. Hence, the performance improves as the $p$ is increased. Secondly, when the battery capacity, $B$, is increased from $2 E_{H}$ to $4 E_{H}$, the performance improves. This is because,
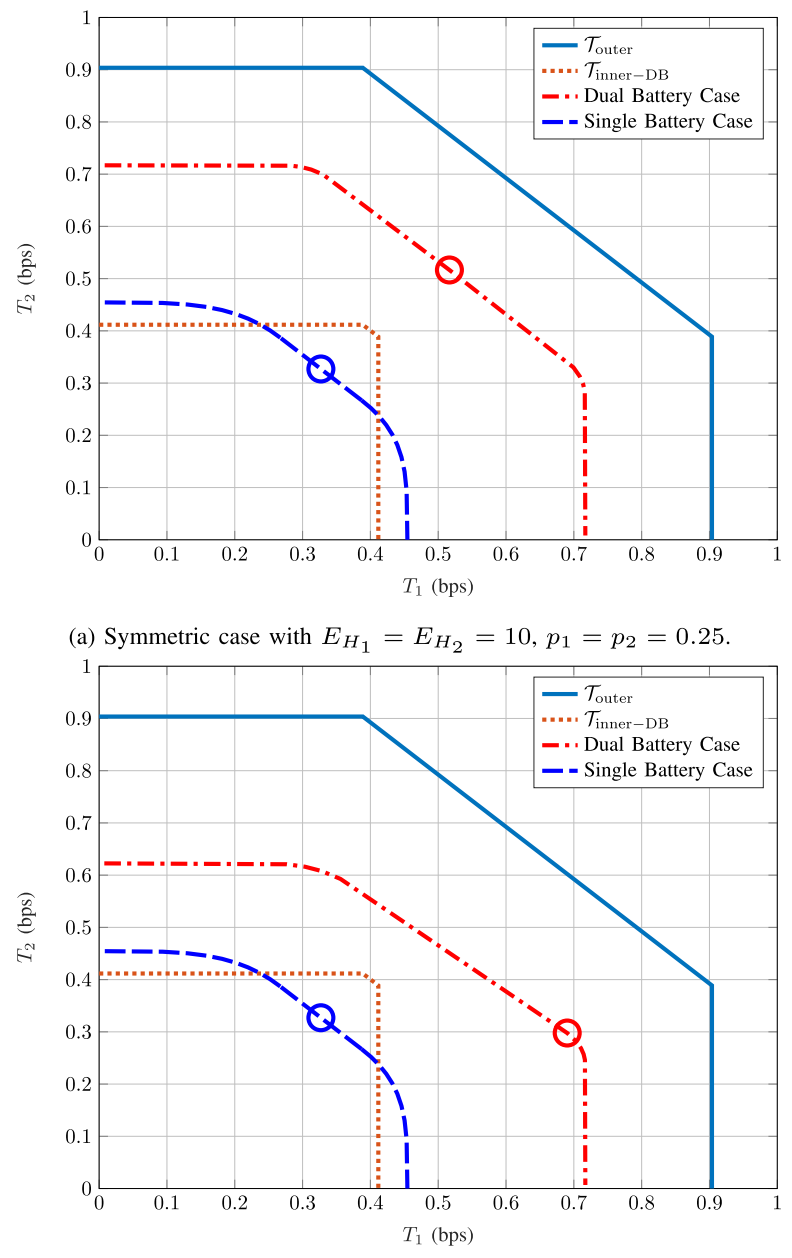

(b) Asymmetric case with $2 E_{H_{1}}=E_{H_{2}}=20, p_{1}=2 p_{2}=0.25$

Fig. 7. Long-term average throughput regions in a two-user MAC with single and dual batteries with $B_{1}, B_{2}=20$ units and $\mu_{1}, \mu_{2}=2.5$ in symmetric and asymmetric cases. Markers show the maximum sum throughput points.

average idle time and the average amount of energy discarded when $B=4 E_{H}$ are significantly lower than that when $B=2 E_{H}$, as seen from Table I and Fig. 6, respectively. Finally, we note that some policies are more robust to burstiness than others. Specifically, performance of the SA-I and SA-II policies are better than that of ONA and SNA policies for all values of $p$. Moreover, the variation of SA-I and SA-II policies with $p$ is less than that of the ONA and SNA policies. Further, the CP policy is more robust than the SNA policy for the chosen values. As expected, the long-term average throughput in the single battery case does not vary with $p$, as $\mu$ is kept fixed.

\section{B. Long-Term Average Throughput Regions in a MAC}

In Fig. 7, we present long-term average throughput regions in a two-user MAC under single battery and dual battery cases for symmetric (Fig. 7a) and asymmetric (Fig. 7b) settings. In both the settings, the battery capacity and the mean value of the harvested energy remain the same with $B_{1}=B_{2}=20$ units and $\mu_{1}=\mu_{2}=2.5$. Further, in the symmetric case, the distribution of the energy arrivals is the same in both the users. 

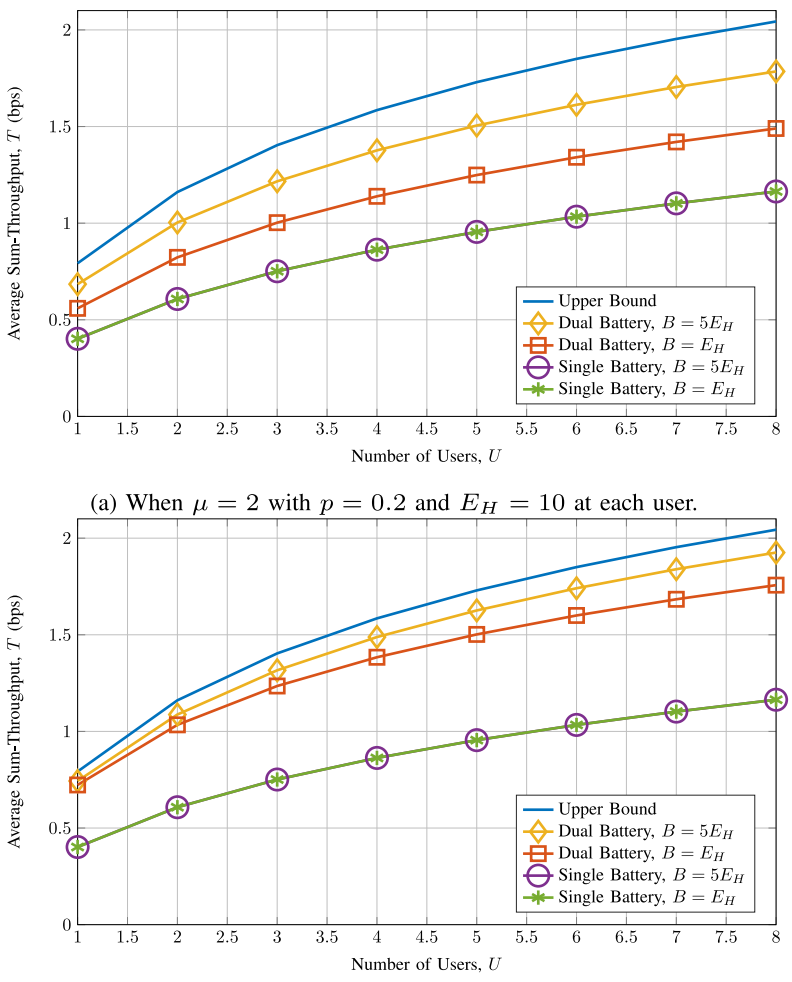

(b) When $\mu=2$ with $p=0.8$ and $E_{H}=2.5$ at each user.

Fig. 8. Variation of the optimal long-term average sum-throughput with $U$.

However, in the asymmetric case, the energy arrivals are more bursty in user 2 . We assume $r_{1}=r_{2}=2$ and $r_{1}=2, r_{2}=1$ in the symmetric and asymmetric cases, respectively. Hence, we have, $\min _{r_{u}} G\left(r_{u}\right)=0.51$ and $\min _{r_{u}} G\left(r_{u}\right)=0.72$ in the symmetric and asymmetric cases, respectively. In both the figures, the largest average throughput region in the dual battery case is significantly larger than that in the single battery case. Moreover, the maximum sum-throughput occurs in the single battery case at $(0.33,0.33)$ in both symmetric and asymmetric cases. However, in the dual battery case, the maximum sum throughput occurs at $(0.52,0.52)$ and $(0.69,0.29)$ in the symmetric and asymmetric cases, respectively. Since both users have the same individual throughputs at the maximum sumthroughput point in the single battery case, it is more fair than the dual battery case in which the individual data throughputs are significantly different at its maximum sum-throughput point. This is because, the optimal power allocation in the single battery case depends only on the mean harvested energy values in both users, unlike in the dual battery case, in which the optimal power allocations depend on the distributions of the harvested energy in both users.

In Fig. 8, we study variation of long-term average sumthroughput, the sum of long-term average throughputs at each user, with the number of users, $U$, when the parameters at all the users are identical with $p=0.2$ and $E_{H}=10$ (see Fig. 8a) and $p=0.8$ and $E_{H}=2.5$ (see Fig. 8b). From the figures, we note that the sum-throughputs are concave function of the number of users, as in the case of an ideal MAC. Moreover, the performance trends are similar to those in the P2P channel. Specifically, as $B$ and $p$ are increased while keeping $\mu$ fixed, the performance increases in the dual battery case, but it remains unchanged in the single battery case.

\section{CONCLUSION}

In this work, we optimized the long-term average throughputs and throughput regions in $\mathrm{P} 2 \mathrm{P}$ and multiple-access communication systems under the single battery and dual battery cases. In order to avoid capacity degradation of the batteries, we applied the cycle constraint, which mandates that a battery must be charged only after it is sufficiently discharged and vice versa. We also applied the half-duplex battery constraint for the charging and discharging processes. We found that the optimal throughput in the dual battery case is significantly higher than that in the single battery case and that the largest throughput region of the proposed multiple access policy in the single battery case is contained within that of the dual battery case. Along the way, we obtained a closed form expression for the optimal power allocation policy in the $\mathrm{P} 2 \mathrm{P}$ channel for the single battery case. For the dual battery case, we first obtained optimal online policy via dynamic programming, followed by non-adaptive and adaptive policies, which do not adapt and adapt power allocations based on battery states, respectively. We then obtained throughput regions in a MAC based on the policies proposed for the P2P channel. We numerically found that the gap between the performance of our suboptimal non-adaptive policy and the unconstrained optimal performance decreases with the battery capacity faster than the inverse of the square root of the battery capacity, in the dual battery case.

\section{APPENDIX A}

PROOF OF LEMMA 1

The problem in (12) is a linear fractional program, which can be transformed into the following equivalent linear program by letting $y_{n}=p_{n} /\left(\sum_{n=1}^{\infty} n p_{n}+\bar{L}_{2 B}\right)$ and $t=$ $\sum_{n=1}^{\infty} n p_{n}+\bar{L}_{2 B}[31]$ :

$$
\begin{aligned}
\underset{y_{1}, y_{2}, \ldots}{\operatorname{maximize}} & \sum_{n=1}^{\infty} y_{n} \frac{n}{2} \log \left(1+\frac{2 B}{n}\right), \\
\text { subject to } & \sum_{n=1}^{\infty} y_{n} \leq t, y_{n} \geq 0, \forall n \in \mathbb{Z}^{+}, \\
& \sum_{n=1}^{\infty} n y_{n}+t \bar{L}_{2 B}=1, t \geq 0 .
\end{aligned}
$$

Now, eliminating $t$ from the equality in (34c), we get the following equivalent problem:

$$
\begin{aligned}
& \underset{y_{n} \geq 0, n \in \mathbb{Z}^{+}}{\operatorname{maximize}} \sum_{n=1}^{\infty} y_{n} \frac{n}{2} \log \left(1+\frac{2 B}{n}\right), \\
& \text { subject to } \sum_{n=1}^{\infty} y_{n}\left(n+\bar{L}_{2 B}\right) \leq 1 .
\end{aligned}
$$

Let $\lambda$ be the dual variable for (35b). Then the equivalent dual linear program of (35) is given by

$$
\underset{\lambda \geq 0}{\operatorname{minimize}} \lambda, \text { subject to } \lambda \geq \frac{\frac{n}{2} \log \left(1+\frac{2 B}{n}\right)}{n+\bar{L}_{2 B}}, \forall n \in \mathbb{Z}^{+} \text {. }
$$


In the above problem, $\lambda$ is constrained only by the maximum value of $\left(\frac{n}{2} \log \left(1+\frac{2 B}{n}\right)\right) /\left(n+\bar{L}_{2 B}\right)$. Hence, we have,

$$
\lambda^{*}=\max _{n \in \mathbb{Z}^{+}} \frac{\frac{n}{2} \log \left(1+\frac{2 B}{n}\right)}{n+\bar{L}_{2 B}},
$$

and due to the strong-duality, we have, $T_{\mathrm{SB}}^{*}=\lambda^{*}$.

\section{APPENDIX B \\ ProOF OF THEOREM 2}

Taking the derivative of the objective function in (14) and equating it to zero, the optimal $\tilde{P}$ must satisfy

$$
\mu+\tilde{P}-(1+\tilde{P}) \log (1+\tilde{P})=0
$$

For $\mu \geq 1$, (38) has only one real solution given by $(\mu-1) /\left(W_{0}(\exp (-1)(\mu-1))\right)-1$, where $W_{0}(\cdot)$ is the principal branch of the Lambert $W$ function [28]. For $0<\mu<1$, (38) has two real solutions, namely, $(\mu-1) /\left(W_{0}(\exp (-1)(\mu-1))\right)-1 \quad$ and $(\mu-1) /\left(W_{-1}(\exp (-1)(\mu-1))\right)-1$, where $W_{-1}$ is the lower branch of the Lambert $\mathrm{W}$ function. Since the former solution is always larger than the latter solution [28], we can always choose the larger value and obtain a higher rate without violating any constraints. Moreover, the latter solution is always negative [28]. Hence, the optimal transmit power is given by (15). Now, noting that $\exp \left(W_{0}(x)\right)=x / W_{0}(x)$, we get $(\mu-1) /\left(W_{0}(\exp (-1)(\mu-1))\right)-1=$ $\exp (1) \exp \left(W_{0}(\exp (-1)(\mu-1))\right)-1$ and we can obtain $\tilde{T}_{\mathrm{SB}}$ by substitution of the optimal $\tilde{P}$ in the objective function.

\section{APPENDix C \\ PROOF OF LEMMA 3}

It is sufficient to consider the following two events, which when occur, the roles of the batteries can be potentially switched: (A) when the charging battery becomes full, (B) when the working battery becomes empty. In all other cases, the roles of the batteries cannot be switched due to the cycle constraint. When both of the above events occur simultaneously in a slot, trivially, it is optimal to switch the roles of the batteries in the same slot. We now consider the scenario when only one of the above events occurs. Without loss of generality, we assume that Battery 1 and Battery 2 are charging and working batteries, respectively, in the slot immediately before the slots where the above events occur.

When only the event A occurs, let $b_{2}>0$ be the amount of energy available in Battery 2. In this case, we can take one of the following two feasible actions: (A-I) wait for Battery 2 to get empty and then switch the roles of the batteries, and (A-II) switch Battery 1 to be the second working battery by drawing a non-zero amount of energy from Battery 1, anytime before Battery 2 gets empty. With A-I, we must suspend EH exactly until Battery 2 becomes empty, whereas, with A-II, we must suspend EH until any one of the batteries becomes empty. Note that with A-II, we are allowed to discharge both the batteries simultaneously at a higher power than the power at which Battery 2 is discharged in A-I. However, such an operation is suboptimal due to the concavity of the $\log (\cdot)$ function. In A-II, when only one of the batteries discharges at a time, the number of slots required for any one of the working batteries to get empty is greater than or equal to the number of slots required for Battery 2 to get empty with action A-I, when transmission power policies in A-I and A-II are identical. ${ }^{1}$ This is because, in A-II, when Battery 1 is switched to be the second working battery, at least one slot is used for drawing a non-zero amount of energy from it. Hence, in the slot when either Battery 1 or Battery 2 become empty with A-II, the amount of energy available in Battery 2 with A-I is greater than or equal to zero and the amount of energy available in Battery 1 with A-I is equal to that in the non-empty battery with A-II. Hence, in the subsequent slots, the achievable throughput with A-I is at least as much as that with A-II.

Similarly, when only the event B occurs, let $b_{1}<B$ be the amount of energy available in Battery 1 . In this case, we can take one of the following two feasible actions: B-I wait for Battery 1 to get full and then switch the roles of the batteries, and B-II switch Battery 2 to be the second charging battery. With B-I, transmission will be suspended until Battery 1 gets full. On the other hand, with B-II, transmission will be suspended until any one of the battery gets full, which will happen strictly after Battery 1 gets full in B-I. In this case, the throughput achieved with B-I will be at least as much as that with B-II. This is because, with action B-I, we can always wait until one of the batteries with action B-II gets full and then start transmission.

Since the throughputs achieved by A-I and B-I are greater than or equal to those in A-II and B-II, respectively, and because there are no other feasible actions, the lemma follows. Note that we have made use of the fact that with the Bernoulli EH model in (1), the amount of energy harvested in an arrival is such that it does not overflow from the charging battery if it was not full in the slot before the energy arrival.

\section{APPENDIX D}

\section{Optimal Offline Policy for the Dual Battery Case}

In the offline policy, the number of slots required to completely charge the battery is known at the start of the current renewal instant, i.e., the realization of $L$ is known. Due to the concavity of $\frac{1}{2} \log (1+P)$ in $P$, it is optimal to transmit with a constant power of $B / L$ over the next $L$ slots after a renewal instant. Hence, the distributions of the length of the renewal period, $C$ and $L$ are identical. Thus, from (19), the optimal long-term average throughput is given by

$$
T_{\text {off }}=\frac{p}{r} \sum_{m=r}^{\infty} \frac{m q_{m}}{2} \log \left(1+\frac{B}{m}\right) .
$$

It is easy to numerically compute the value of $T_{\text {off }}$.

\footnotetext{
${ }^{1}$ From the throughput perspective, transmitting with power $x$ in slot 1 and $y$ is slot 2 is identical to transmitting with power $y$ in slot 1 and $x$ in slot 2 as the sum-throughput achieved in both the cases is equal to $\frac{1}{2} \log (1+x)+$ $\frac{1}{2} \log (1+y)$. Hence, any power allocation policy achievable with A-II is also achievable with A-I, possibly with a different order of transmit powers.
} 


\section{APPENDIX E}

\section{ProOF of THEOREM 4}

The Lagrangian of (26) is given by

$$
\begin{aligned}
H= & -\sum_{i=1}^{\infty} \bar{F}_{i-1}(r, p) \frac{p}{2 r} \log \left(1+\tilde{P}_{i}\right)+\lambda\left(\sum_{i=1}^{\infty} \tilde{P}_{i}-B\right) \\
& -\sum_{i=1}^{\infty} \omega_{i} \tilde{P}_{i},
\end{aligned}
$$

where $\lambda, \omega_{i} \geq 0$ are Lagrange multipliers. We now have the following stationarity condition: For all $i \in\{1,2, \ldots\}$,

$$
\frac{\partial H}{\partial \tilde{P}_{i}}=-\frac{p \bar{F}_{i-1}(r, p)}{2 r \ln 2\left(1+\tilde{P}_{i}\right)}+\lambda-\omega_{i}=0 .
$$

Further, the complementary slackness condition, $\omega_{i} \tilde{P}_{i}=0$, must be satisfied at the optimal solution. Hence, when $\tilde{P}_{i}>0$, we must have, $\omega_{i}=0$ and from (40), we get

$$
\tilde{P}_{i}=\frac{p \bar{F}_{i-1}(r, p)}{2 r \lambda \ln 2}-1, \quad i=1,2, \ldots
$$

The optimal $\lambda$ can be found to be $\lambda^{*}=$ $\left(p \sum_{i=1}^{M} \bar{F}_{i-1}(r, p)\right) /(2 r \ln 2(B+M))$ from the total energy constraint, where $M$ is the last slot in which $\tilde{P}_{i}>0$. From (41) and the expression for $\lambda^{*}$, we find that $M$ is the largest $m$ that satisfies $\sum_{i=1}^{m} \bar{F}_{i-1}(r, p) \leq(B+m) F_{m}(r, p)$. Now, noting that $\bar{F}_{i-1}(r, p)=1$ for $i \leq r$, we obtain (27).

\section{APPENDIX F \\ Proof OF THEOREM 5}

We prove the theorem along the lines in [14, Proposition 3] in the following.

$$
\begin{aligned}
T_{\text {SNA }} & =\frac{\mathbb{E}\left[\sum_{i=1}^{L} \frac{1}{2} \log \left(1+\tilde{P}_{i}\right)\right]}{\mathbb{E}[L]} \\
& =\frac{\mathbb{E}\left[\sum_{i=1}^{L} \frac{1}{2} \log \left(1+\frac{B p}{r} \sum_{m=i}^{\infty} q_{m}\right)\right]}{\mathbb{E}[L]}, \\
& \stackrel{\text { (a) }}{\geq} \frac{\mathbb{E}\left[\sum_{i=1}^{L} \frac{1}{2} \log \left(1+\frac{B p}{r}\right)+\sum_{i=1}^{L} \frac{1}{2} \log \left(\sum_{m=i}^{\infty} q_{m}\right)\right]}{\mathbb{E}[L]}, \\
& =\frac{1}{2} \log \left(1+\frac{B p}{r}\right)+\frac{p}{r} \sum_{i=1}^{\infty}\left(\sum_{m=i}^{\infty} q_{m}\right) \frac{1}{2} \log \left(\sum_{m=i}^{\infty} q_{m}\right) \\
& \geq \frac{1}{2} \log (1+\mu)-G(r),
\end{aligned}
$$

where (a) is because $\log (1+\alpha x) \geq \log (1+x)+$ $\log (\alpha)$ for $0<\alpha \leq 1$ and we define $G(r) \triangleq$ $\max _{p}(-p / r) \sum_{i=1}^{\infty}\left(\sum_{m=i}^{\infty} q_{m}\right) \frac{1}{2} \log \left(\sum_{m=i}^{\infty} q_{m}\right)$.

\section{REFERENCES}

[1] R. V. Bhat, M. Motani, C. R. Murthy, and R. Vaze, "Energy harvesting communications with batteries having full-cycle constraints," in Proc. IEEE ICC, Shanghai, China, May 2019, pp. 1-6.

[2] Q. Wu, G. Y. Li, W. Chen, D. W. K. Ng, and R. Schober, "An overview of sustainable green $5 \mathrm{G}$ networks," IEEE Wireless Commun., vol. 24, no. 4, pp. 72-80, Aug. 2017.

[3] S. Ulukus et al., "Energy harvesting wireless communications: A review of recent advances," IEEE J. Sel. Areas Commun., vol. 33, no. 3, pp. 360-381, Mar. 2015.
[4] R. V. Bhat, M. Motani, and T. J. Lim, "Energy harvesting communication using finite-capacity batteries with internal resistance," IEEE Trans. Wireless Commun., vol. 16, no. 5, pp. 2822-2834, May 2017.

[5] S. Reddy and C. R. Murthy, "Dual-stage power management algorithms for energy harvesting sensors," IEEE Trans. Wireless Commun., vol. 11, no. 4, pp. 1434-1445, Apr. 2012.

[6] S. Satpathi, R. Nagda, and R. Vaze, "Optimal offline and competitive online strategies for transmitter-receiver energy harvesting," IEEE Trans. Inf. Theory, vol. 62, no. 8, pp. 4674-4695, Aug. 2016.

[7] R. A. Huggins, "Mechanism of the memory effect in 'Nickel' electrodes," Solid-State Ionics, vol. 177, nos. 26-32, pp. 2643-2646, 2006

[8] T. Sasaki, Y. Ukyo, and P. Novák, "Memory effect in a lithium-ion battery," Nat. Mater., vol. 12, no. 6, pp. 569-575, Jun. 2013.

[9] G. M. Siddesh, G. C. Deka, K. G. Srinivasa, and L. M. Patnaik, CyberPhysical Systems: A Computational Perspective. Boca Raton, FL, USA: CRC Press, 2015.

[10] N. Michelusi, L. Badia, R. Carli, L. Corradini, and M. Zorzi, "Energy management policies for harvesting-based wireless sensor devices with battery degradation," IEEE Trans. Commun., vol. 61, no. 12, pp. 4934-4947, Dec. 2013.

[11] R. Valentini, M. Levorato, and F. Santucci, "Optimal aging-aware channel access and power allocation for battery-powered devices with radio frequency energy harvesting," IEEE Trans. Commun., vol. 66, no. 11, pp. 5773-5787, Nov. 2018.

[12] M. Mendil, A. De Domenico, V. Heiries, R. Caire, and N. Hadjsaid, "Battery-aware optimization of green small cells: Sizing and energy management," IEEE Trans. Green Commun. Netw., vol. 2, no. 3, pp. 635-651, Sep. 2018.

[13] J. Yang and J. Wu, "Optimal transmission for energy harvesting nodes under battery size and usage constraints," in Proc. IEEE ISIT, Jun. 2017, pp. 819-823.

[14] D. Shaviv and A. Özgür, "Universally near optimal online power control for energy harvesting nodes," IEEE J. Sel. Areas Commun., vol. 34, no. 12 , pp. 3620-3631, Dec. 2016.

[15] H. A. Inan and A. Özgür, "Online power control for the energy harvesting multiple access channel," in Proc. IEEE WiOpt, May 2016, pp. 1-6.

[16] S. Kapoor and S. R. B. Pillai, "Distributed scheduling schemes in energy harvesting multiple access," IEEE Wireless Commun. Lett., vol. 6, no. 1, pp. 54-57, Feb. 2017.

[17] A. Baknina and S. Ulukus, "Online policies for multiple access channel with common energy harvesting source," in Proc. IEEE ISIT, Jul. 2016, pp. $2739-2743$.

[18] A. Arafa, A. Baknina, and S. Ulukus, "Online fixed fraction policies in energy harvesting communication systems," IEEE Trans. Wireless Commun., vol. 17, no. 5, pp. 2975-2986, May 2018.

[19] D. Shaviv and A. Özgür, "Online power control for block i.i.d. Bernoulli energy harvesting channels," in Proc. IEEE WCNCW, Mar. 2017, pp. 1-6.

[20] A. Kazerouni and A. Özgür, "Optimal online strategies for an energy harvesting system with Bernoulli energy recharges," in Proc. IEEE WiOpt, May 2015, pp. 235-242.

[21] N. Michelusi, K. Stamatiou, and M. Zorzi, "On optimal transmission policies for energy harvesting devices," in Proc. IEEE ITA, Feb. 2012, pp. 249-254.

[22] S. Yin, E. Zhang, J. Li, L. Yin, and S. Li, "Throughput optimization for self-powered wireless communications with variable energy harvesting rate," in Proc. IEEE WCNC, Apr. 2013, pp. 830-835.

[23] S. Luo, R. Zhang, and T. J. Lim, "Optimal save-then-transmit protocol for energy harvesting wireless transmitters," IEEE Trans. Wireless Commun., vol. 12, no. 3, pp. 1196-1207, Mar. 2013.

[24] O. Ozel and S. Ulukus, "Achieving AWGN capacity under stochastic energy harvesting," IEEE Trans. Inf. Theory, vol. 58, no. 10, pp. 6471-6483, Oct. 2012.

[25] R. Srivastava and C. E. Koksal, "Basic performance limits and tradeoffs in energy-harvesting sensor nodes with finite data and energy storage," IEEE/ACM Trans. Netw., vol. 21, no. 4, pp. 1049-1062, Aug. 2013.

[26] R. Rajesh, P. K. Deekshith, and V. Sharma, "Capacity of a Gaussian MAC with energy harvesting transmit nodes," in Proc. IEEE ITA, Feb. 2012, pp. 338-343.

[27] R. G. Gallager, Stochastic Processes: Theory for Applications. Cambridge, U.K.: Cambridge Univ. Press, 2014.

[28] R. M. Corless, G. H. Gonnet, D. E. G. Hare, D. J. Jeffrey, and D. E. Knuth, "On the Lambert $W$ function," in Advances in Computational Mathematics. Amsterdam, The Netherlands: Baltzer Sci., 1996, pp. 329-359. 
[29] A. Aprem, C. R. Murthy, and N. B. Mehta, "Transmit power control policies for energy harvesting sensors with retransmissions," IEEE $J$. Sel. Topics Signal Process., vol. 7, no. 5, pp. 895-906, Oct. 2013.

[30] K. Shen and W. Yu, "Fractional programming for communication systems-Part I: Power control and beamforming," IEEE Trans. Signal Process., vol. 66, no. 10, pp. 2616-2630, May 2018.

[31] S. Boyd and L. Vandenberghe, Convex Optimization. New York, NY, USA: Cambridge Univ. Press, 2004.

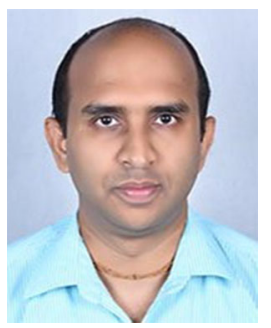

Rajshekhar Vishweshwar (R. V.) Bhat (S'15M'19) received the B.Tech. degree in electrical and communication engineering from the National Institute of Technology, Surathkal, India, in 2010, the M.Tech. degree from the Indian Institute of Technology, Kanpur, India, in 2012, and the $\mathrm{Ph} . \mathrm{D}$. degree from the Electrical and Computer Engineering Department, National University of Singapore, Singapore, in 2019. From 2012 to 2014, he was a Design Engineer with Analog Devices Inc., Bengaluru, India. He was also a Visiting Fellow with TIFR Mumbai, India. He is currently an Assistant Professor with the Department of Electrical Engineering, Indian Institute of Technology, Dharwad, India. His research interests are in the areas of energy harvesting and low-latency communications, and applications of deep learning techniques for wireless communications.

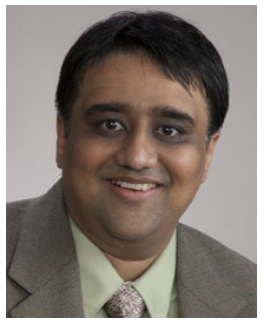

Mehul Motani (S'92-M'01-SM'15-F'18) received the B.E. degree in electrical and computer engineering from Cooper Union, New York, NY, USA, the M.S. degree in electrical and computer engineering from Syracuse University, Syracuse, NY, USA, and the Ph.D. degree in electrical and computer engineering from Cornell University, Ithaca, NY, USA.

He was a Visiting Fellow with Princeton University. He is currently an Associate Professor with the Electrical and Computer Engineering Department, National University of Singapore (NUS) and a Visiting Research Collaborator with Princeton University. He was also a Research Scientist with the Institute for Infocomm Research, Singapore, for three years and a Systems Engineer with Lockheed Martin, Syracuse, for over four years. His research interests include information theory and coding, machine learning, biomedical informatics, wireless and sensor networks, and the Internet of Things.

Dr. Motani was the recipient of the Intel Foundation Fellowship for his Ph.D. research, the NUS Annual Teaching Excellence Award, the NUS Faculty of Engineering Innovative Teaching Award, and the NUS Faculty of Engineering Teaching Honors List Award. He actively participates in the Institute of Electrical and Electronics Engineers (IEEE) and the Association for Computing Machinery (ACM). He has served as the Secretary of the IEEE Information Theory Society Board of Governors. He has served as an Associate Editor for the IEEE TRANSACTIONS ON INFORMATION THEORY and the IEEE TRANSACTIONS ON COMMUNiCATIONS. He has also served on the Organizing and Technical Program Committees of numerous IEEE and ACM conferences.

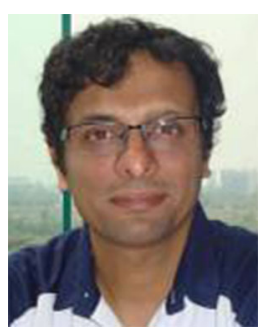

Chandra R. Murthy received the B.Tech. degree in electrical engineering from the Indian Institute of Technology Madras, Chennai, India, in 1998, the M.S. degree in electrical and computer engineering from Purdue University, West Lafayette, IN, USA, in 2000, and the Ph.D. degree in electrical and computer engineering from the University of California, San Diego, CA, USA, in 2006.

From 2000 to 2002, he was an Engineer with Qualcomm Inc., San Jose, USA, where he researched on WCDMA baseband transceiver design and 802.11b baseband receivers. From 2006 to 2007, he was a Staff Engineer with Beceem Communications Inc., Bengaluru, India, on advanced receiver architectures for the 802.16e Mobile WiMAX Standard. He is currently a Professor with the Department of Electrical Communication Engineering, Indian Institute of Science, Bengaluru. He has over 50 journal papers and over 80 conference papers to his credit. His research interests are in the areas of energy harvesting communications, multiuser MIMO systems, and sparse signal recovery techniques applied to wireless communications. He was a recipient of the Best Paper Award in the Communications Track at NCC 2014 for his paper and a paper coauthored with his student won the Student Best Paper Award at the IEEE ICASSP 2018. He was an Associate Editor of the IEEE SignAL PROCESSING LeTTERS from 2012 to 2016. He is an Elected Member of the IEEE SPCOM Technical Committee from 2014 to 2016, and has been re-elected for the terms 2017 to 2019. He is a Past Chair of the IEEE Signal Processing Society, Bangalore Chapter. He is currently serving as an Associate Editor for the IEEE TRANSACTIONS ON SIGNAL PROCESSING and the IEEE TRANSACTIONS ON INFORMATION THEORY, and as an Editor for the IEEE TRANSACTIONS ON COMMUNICATIONS.

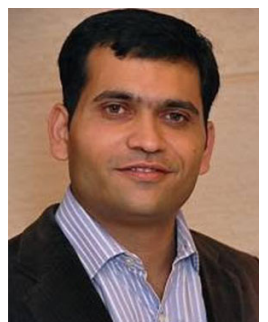

Rahul Vaze (M'09) received the B.E. degree in electronics engineering from the Madhav Institute of Technology and Science, Gwalior, India, in 2002, the M.E. degree in telecommunications from the Indian Institute of Science, Bengaluru, India, in 2004, and the Ph.D. degree from the University of Texas, Austin, in 2009. Since October 2009, he has been a Reader with the School of Technology and Computer Science, Tata Institute of Fundamental Research, Mumbai, India. He has authored the book entitled Random Wireless Networks (Cambridge University Press, 2015). His research interests are in multiple antenna communication, ad hoc networks, and combinatorial resource allocation. He was a recipient of the Indian National Science Academy's Young Scientist Award for the year 2013 and the Indian National Academy of Engineering's Young Engineer Award for the year 2013. He was a co-recipient of the EURASIP Best Paper Award in 2010 for the Journal of Wireless Communication and Networking. 